TRANSACTIONS OF THE

AMERICAN MATHEMATICAL SOCIETY

Volume 351, Number 5, Pages 1947-1974

S 0002-9947(99)02160-

Article electronically published on January 27, 1999

\title{
LOCAL TOMOGRAPHY WITH NONSMOOTH ATTENUATION
}

\author{
A. I. KATSEVICH
}

\begin{abstract}
Local tomography for the Radon transform with nonsmooth attenuation is proposed and justified. The main theoretical tool is analysis of singularities of pseudodifferential operators with nonsmooth symbols. Results of numerical testing of local tomography are presented.
\end{abstract}

\section{INTRODUCTION}

The theory of Single Photon Emission Computed Tomography (SPECT), which is widely used in nuclear medicine, is based on the attenuated Radon transform:

$$
\begin{gathered}
\hat{f}^{(\Phi)}(\theta, p)=\int_{-\infty}^{\infty} f\left(p \Theta+t \Theta^{\perp}\right) \exp \left(-\int_{t}^{\infty} \mu\left(p \Theta+s \Theta^{\perp}\right) d s\right) d t \\
\Theta=(\cos \theta, \sin \theta), \Theta^{\perp}=(-\sin \theta, \cos \theta) .
\end{gathered}
$$

Here $f(x)$ is the density of some radioactive isotope inside a patient, and the coefficient $\mu(x)$ characterizes attenuating properties of tissues. The coefficient $\mu(x)$ is assumed to be known, and $f$ is to be determined from the data $\hat{f}^{(\Phi)}(\theta, p), \theta \in$ $[0,2 \pi), p \in \mathbb{R}$. If the attenuating properties of the medium can be neglected, the reconstruction procedure is equivalent to the classical Radon transform inversion. In most cases attenuation cannot be neglected [De, p.17], and the problem of inverting the transform (1.1) becomes very complicated. Explicit inversion formulas exist only in the case when $\mu(x) \equiv$ const [TM] or when $\mu$ does not depend on $x$ and depends only on the direction in which radiation propagates $[\mathrm{KS}]$. In general, when $\mu$ depends on $x$, no inversion formula is known.

It turns out that although exact inversion of (1.1) is not possible, one can recover the singularities of $f$. The basic idea consists of computing not $f$, but $\mathcal{B} f$, where $\mathcal{B}$ is an elliptic pseudodifferential operator (PDO). Moreover, if $\mathcal{B}$ is appropriately chosen, calculation of $\mathcal{B} f$ is local. To compute $\mathcal{B} f$ at a point $x$ one uses the tomographic data $\hat{f}^{(\Phi)}(\theta, p)$ for $\theta \in[0,2 \pi)$ and $|p-\Theta \cdot x|<\epsilon$, where $\epsilon>0$ can be chosen arbitrarily small. If the order of $\mathcal{B}$ is positive (in practice, the order of $\mathcal{B}$ equals 1), the singularities of $f$ are better visible in $\mathcal{B} f$. A group of methods based on computing $\mathcal{B} f$ is known as local tomography. The first local tomography formulas were proposed by Vainberg et al. [VKK] and Smith and Keinert [SK] for the classical Radon transform. Further investigation of local tomography is contained in $[\mathrm{FRS}],[\mathrm{FFRS}],[\mathrm{K} 2],[\mathrm{KR} 1],[\mathrm{KR} 2],[\mathrm{KR} 3],[\mathrm{R} 1],[\mathrm{R} 2],[\mathrm{R} 3],[\mathrm{RK}]$. An approach to local inversion of the classical Radon transform based on wavelets is proposed in

Received by the editors May 30, 1996 and, in revised form, November 13, 1996.

1991 Mathematics Subject Classification. Primary 35S99, 44A12, 65R10, 92C55.

(C)1999 American Mathematical Society 
[BW]. Later, local tomography was developed for the generalized Radon transform $[\mathrm{KLM}],[\mathrm{K} 1],[\mathrm{RK}]$.

Usually one assumes that the attenuation coefficient $\mu(x)$ is a $C^{\infty}$ function of $x$, because this assumption allows one to use the classical theory of PDO (see e.g. [Shu]) for the analysis of the tomographic reconstruction [Be], [K1], [KLM], [RK]. This, however, appears to be very restrictive, because in many cases the attenuation is not smooth: as an example, one can think of a bone/soft tissue interface, where the attenuation coefficient $\mu(x)$ experiences a jump. It appears that no results are known in the literature about the behavior of tomographic reconstructions in the case of nonsmooth attenuation. Although many strong results on PDO whose symbols have limited smoothness have been obtained (see e.g. [BR], [GU], [Ma1], [Ma2], [MU], [Ta], and references therein), these results cannot be applied to the analysis of problems which occur in emission tomography because of the specific nature of the singularities of the resulting symbols. For example, one frequently assumes that the symbols are $C^{\infty}$-smooth with respect to the dual variable $\xi$ (see e.g. [Ta]), but the symbols which will be considered below are not smooth in $\xi$.

The main purpose of this paper is to develop a theoretical basis of local tomography for the Radon transform with nonsmooth attenuation, and to demonstrate by numerical experiments the validity of the theory.

In Section 2 we introduce a local tomography formula and state the main theorem. A proof of this theorem is based on four lemmas, which are stated and proved in Sections 3 and 4. Application of the result to conventional tomographic reconstruction, which ignores attenuation, is described in Section 5. There exist several methods for attenuation correction in emission tomography (see e.g. [Kun] and references therein), but they are, for the most part, iterative in nature and do not always guarantee convergence. In particular, the algorithm in [Kun] cannot be used in the case of piecewise smooth $\mu$, which is considered here. A simple algorithm for the attenuation correction, which allows one to find correct values of jumps of $f$, is described in Section 5. Using the theorem in Section 2 and results in [K1], we describe an algorithmic implementation of local tomography in Section 6. Results

of numerical testing of the algorithms are presented in Section 7. Section 8 contains auxiliary results needed for the proof of the main theorem.

\section{Statement of the main Result}

Consider the attenuated Radon transform (1.1), which can be represented as follows:

$$
\hat{f}^{(\Phi)}(\theta, p)=\int_{\mathbb{R}^{2}} f(x) \Phi(x, \theta) \delta(p-\Theta \cdot x) d x,
$$

where

$$
\Phi(x, \theta)=\exp \left\{-\int_{0}^{\infty} \mu\left(x+t \Theta^{\perp}\right) d t\right\},
$$

and $\mu(x)$ is the attenuation coefficient. In (2.1), (2.2), and everywhere below, the variables $\theta, \Theta$, and $\Theta^{\perp}$ are always related as follows: $\Theta=(\cos \theta, \sin \theta)$ and $\Theta^{\perp}=(-\sin \theta, \cos \theta)$.

Let us suppose that $\mu$ is piecewise smooth, and denote $\Gamma=\operatorname{singsupp} \mu$. We suppose that $\Gamma$ is a union of finitely many smooth nonintersecting curves. Assumptions about $\mu$ and $\Gamma$ are formulated more precisely in Theorem 1 , below. 
Introduce the local tomography function $\tilde{f}_{\Lambda}^{(\Phi)}$ by the formula

$$
\tilde{f}_{\Lambda}^{(\Phi)}(x)=-\frac{1}{4 \pi} \int_{0}^{2 \pi} \hat{f}_{, p p}^{(\Phi)}(\theta, \Theta \cdot x) d \theta
$$

where $\hat{f}_{, p p}^{(\Phi)}=\partial^{2} \hat{f}^{(\Phi)} / \partial p^{2}$. The function $\tilde{f}_{\Lambda}^{(\Phi)}$ was introduced for the generalized Radon transform with smooth weight in [KLM]. Substituting (2.1) into (2.3) and using the well-known oscillatory integral $\delta(t)=(2 \pi)^{-1} \int_{-\infty}^{\infty} \exp (i \lambda t) d \lambda$, we get

$$
\tilde{f}_{\Lambda}^{(\Phi)}(x)=\frac{1}{(2 \pi)^{2}} \int_{\mathbb{R}^{2}} \int_{\mathbb{R}^{2}}|\xi| b(y, \xi /|\xi|) f(y) e^{-i \xi \cdot(x-y)} d y d \xi,
$$

where

$$
b(y, \theta)=\frac{1}{2}\left[\exp \left\{-\int_{0}^{\infty} \mu\left(y+t \Theta^{\perp}\right) d t\right\}+\exp \left\{-\int_{0}^{\infty} \mu\left(y-t \Theta^{\perp}\right) d t\right\}\right] .
$$

It is easy to check that $b(y, \theta)=b(y, \theta+\pi)$. Using this property and writing the integral in (2.4) in the polar coordinate system, we get

$$
\tilde{f}_{\Lambda}^{(\Phi)}(x)=\frac{2}{(2 \pi)^{2}} \operatorname{Re}\left\{\int_{0}^{\infty} \int_{\frac{1}{2} S^{1}} \int_{\mathbb{R}^{2}} b(y, \theta) f(y) e^{-i \sigma \Theta \cdot(x-y)} d y d \theta \sigma^{2} d \sigma\right\},
$$

where $\frac{1}{2} S^{1}$ is an arbitrary half of the unit sphere $S^{1}$.

Let us introduce the following notation. For $x \in \Gamma, L_{\Gamma}(x)$ denotes a line tangent to $\Gamma$ at $x$. Given any domain $D \subset \mathbb{R}^{2}$, we denote also

$$
\Gamma_{D}:=\overline{\left\{x \in \Gamma: D \cap L_{\Gamma}(x) \neq \varnothing\right\}} \text {. }
$$

In other words, $\Gamma_{D}$ is the closure of the set of points of $\Gamma$ such that lines tangent to $\Gamma$ at these points intersect $D$ (see Figure 3.1, below). Given a set $A \subset \mathbb{R}^{2}, U_{\epsilon}(A)$ denotes an $\epsilon$-neighborhood of $A$. In particular, $U_{\epsilon}\left(x_{0}\right)$ is a ball with center $x_{0}$ and radius $\epsilon>0$.

Theorem 1. Suppose that $f$ and $\mu$ can be represented in the form

$$
f(x)=\sum_{k} f_{k}(x) \chi_{f, k}(x), \mu(x)=\sum_{k} \mu_{k}(x) \chi_{\mu, k}(x),
$$

where the sums are finite, $f_{k}, \mu_{k} \in C^{\infty}\left(\mathbb{R}^{2}\right)$, and $\chi_{f, k}, \chi_{\mu, k}$ are the characteristic functions of the bounded domains $D_{f, k}, D_{\mu, k}$, respectively. Boundaries of the domains $D_{f, k}$ are piecewise smooth, and boundaries of the domains $D_{\mu, k}$ are smooth. Suppose that

$$
\partial D_{\mu, k_{1}} \cap \partial D_{\mu, k_{2}}=\varnothing, k_{1} \neq k_{2},
$$

and for any pair $k, j$ either $\partial D_{f, k} \cap \partial D_{\mu, j}=\varnothing$ or $\partial D_{f, k}=\partial D_{\mu, j}$. In the latter case, the boundary $\partial D_{f, k}$ is smooth. Denote $S=\bigcup_{k} \partial D_{f, k}$ and $\Gamma=\bigcup_{k} \partial D_{\mu, k}$. Then

$$
\operatorname{singsupp} \tilde{f}_{\Lambda}^{(\Phi)} \subset S \cup \Gamma_{D} \cup\left(\bigcup_{j} L_{j}\right),
$$

where $L_{j}$ are the lines such that

(a) $L_{j} \cap D \neq \varnothing$,

(b) $L_{j}$ is tangent to $\Gamma$,

and at least one of the following conditions holds: 
(c1) $L_{j}$ is tangent to $S \cup \Gamma$ at more than one point, or

(c2) the radius of curvature of $\Gamma$ at $\Gamma \cap L_{j}$ equals infinity.

Fix any $x_{0} \in S, x_{0} \notin \Gamma \cup\left(\bigcup_{j} L_{j}\right)$, such that $S$ is smooth in a neighborhood of $x_{0}$. Let $n\left(x_{0}\right)$ be a unit vector perpendicular to $S$ at $x_{0}$, and $D_{f}\left(x_{0}\right)$ be the jump of $f$ at $x_{0}$ :

$$
D_{f}\left(x_{0}\right)=\lim _{h \rightarrow+0}\left[f\left(x_{0}+h n\left(x_{0}\right)\right)-f\left(x_{0}-h n\left(x_{0}\right)\right)\right] .
$$

Then

$$
\tilde{f}_{\Lambda}^{(\Phi)}(x)=\frac{b\left(x_{0}, n\left(x_{0}\right)\right)}{\pi} \operatorname{Im}\left\{\int_{0}^{\infty} \Psi_{f}(t, x) e^{i t h} d t\right\}, \quad x=x_{0}+h n\left(x_{0}\right),
$$

where $b$ is defined by (2.5), and $\Psi_{f}(t, x) \in C^{\infty}\left([0, \infty) \times U_{\epsilon}\left(x_{0}\right)\right)$ for some $\epsilon>0$. Moreover, $\Psi_{f}$ admits the asymptotic expansion

$$
\Psi_{f}(t, x) \sim D_{f}\left(x_{0}\right)+\sum_{k \geq 1} \frac{d_{k}(x)}{t^{k}}, \quad t \rightarrow \infty, d_{k} \in C^{\infty}\left(U_{\epsilon}\left(x_{0}\right)\right),
$$

which can be differentiated with respect to $t$ and $x$.

Fix any $x_{0} \in \Gamma_{D}, x \notin S \cup\left(\bigcup_{j} L_{j}\right)$. Let $n\left(x_{0}\right)$ be a unit vector perpendicular to $\Gamma$ at $x_{0}$, and $D_{\mu}\left(x_{0}\right)$ be the jump of $\mu$ at $x_{0}$ :

$$
D_{\mu}\left(x_{0}\right)=\lim _{h \rightarrow+0}\left[\mu\left(x_{0}+h n\left(x_{0}\right)\right)-\mu\left(x_{0}-h n\left(x_{0}\right)\right)\right] .
$$

Then

$$
\tilde{f}_{\Lambda}^{(\Phi)}(x)=-\frac{A\left(x_{0}, \mu, f\right)}{2 \pi} \operatorname{Im}\left\{\int_{0}^{\infty} \Psi_{\mu}(t, x) e^{i t h} d t\right\}, \quad x=x_{0}+h n\left(x_{0}\right),
$$

where

$$
\begin{aligned}
A\left(x_{0}, \mu, f\right)= & \int_{-\infty}^{0} \exp \left[-\int_{t}^{\infty} \mu\left(x_{0}+s n\left(x_{0}\right)^{\perp}\right) d s\right] f\left(x_{0}+\operatorname{tn}\left(x_{0}\right)^{\perp}\right) d t \\
& +\int_{0}^{\infty} \exp \left[-\int_{-\infty}^{t} \mu\left(x_{0}+s n\left(x_{0}\right)^{\perp}\right) d s\right] f\left(x_{0}+\operatorname{tn}\left(x_{0}\right)^{\perp}\right) d t,
\end{aligned}
$$

and $\Psi_{\mu}(t, x) \in C^{\infty}\left([0, \infty) \times U_{\epsilon}\left(x_{0}\right)\right)$ for some $\epsilon>0$. Moreover, $\Psi_{\mu}$ admits the asymptotic expansion

$$
\Psi_{\mu}(t, x) \sim D_{\mu}\left(x_{0}\right)+\sum_{k \geq 1} \frac{d_{k}(x)}{t^{k}}, \quad t \rightarrow \infty, d_{k} \in C^{\infty}\left(U_{\epsilon}\left(x_{0}\right)\right),
$$

which can be differentiated with respect to $t$ and $x$.

Fix any $x_{0} \in S \cap \Gamma, x \notin \bigcup_{j} L_{j}$. Then

$$
\begin{aligned}
\tilde{f}_{\Lambda}^{(\Phi)}(x)= & \frac{b\left(x_{0}, n\left(x_{0}\right)\right)}{\pi} \operatorname{Im}\left\{\int_{0}^{\infty} \Psi_{f}(t, x) e^{i t h} d t\right\} \\
& -\frac{A\left(x_{0}, \mu, f\right)}{2 \pi} \operatorname{Im}\left\{\int_{0}^{\infty} \Psi_{\mu}(t, x) e^{i t h} d t\right\}, \\
x= & x_{0}+h n\left(x_{0}\right),
\end{aligned}
$$

where the functions $\Psi_{f}$ and $\Psi_{\mu}$ have the same properties as above. 


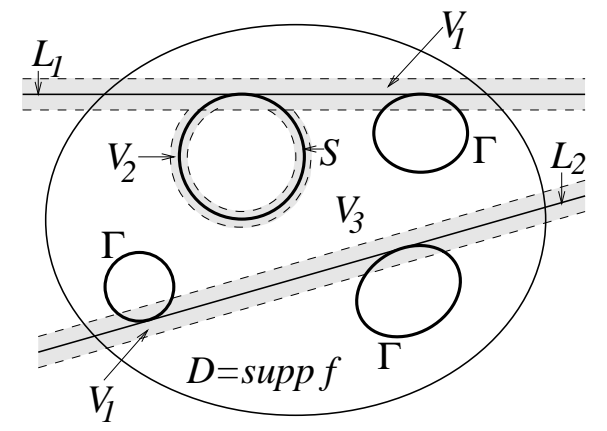

Figure 2.1. Illustration for Theorem 1. Not all the lines $L_{j}$ are shown.

Remark 1. Basically, Theorem 1 asserts that the discontinuities of $\mu$ do not interfere with the discontinuities of $f$ at almost all points $x \in S \backslash \Gamma$. Moreover, as equations (2.9) and (2.10) show, the leading singular term of the local tomography function $\tilde{f}_{\Lambda}^{(\Phi)}$ in a neighborhood of $S$ depends explicitly on values of the jump of $f$ across $S$. Therefore, the latter can be recovered in the same fashion as in the case of smooth attenuation (see Sections 6 and 7 below). Numerical experiments presented in Section 7 confirm this conclusion and show that the lines $L_{j}$ do not cause considerable distortions in local tomography images. However, theoretical analysis of the behavior of $\tilde{f}_{\Lambda}^{(\Phi)}$ in a neighborhood of $L_{j}$ remains an open problem at present.

Remark 2. Equations (2.9)-(2.14) imply that if $D_{f}\left(x_{0}\right) \neq 0$ at any $x_{0} \in S$, and $A\left(x_{0}, \mu, f\right), D_{\mu}\left(x_{0}\right) \neq 0$ at any $x_{0} \in \Gamma_{D}$, then $S \cup \Gamma_{D} \subset \operatorname{singsupp} \tilde{f}_{\Lambda}^{(\Phi)}$.

Proof of Theorem 1. Given a function $f$, let $\tilde{f}_{\Lambda}^{(\Phi)}[f]$ denote the local tomography function corresponding to $f$. Fix sufficiently small $\epsilon, \delta>0$. Define three domains:

$$
V_{1}=U_{\epsilon}\left(\bigcup_{j} L_{j}\right), V_{2}=U_{\delta}(S) \backslash V_{1}, V_{3}=\operatorname{supp} f \backslash\left(V_{1} \cup V_{2}\right),
$$

(see Figure 2.1) and three functions $\chi_{m} \in C_{0}^{\infty}\left(U_{r}\left(V_{m}\right)\right), r=\min (\epsilon, \delta) / 2, m=1,2,3$, such that $\chi_{m}=1$ on $V_{m}$ and $\sum_{m=1}^{3} \chi_{m}(x)=1$ if $x \in \operatorname{supp} f$. Denote $f_{m}=\chi_{m} f$.

The function $f_{2}$ is piecewise smooth. According to the assumptions in Theorem 1 and by construction, there is no line $L$ which satisfies conditions (a)-(c) formulated in Theorem 1 with $D=\operatorname{supp} f_{2}$. Using a partition of unity, represent $f_{2}$ as follows: $f_{2}=\sum_{k} f_{2, k}$, where each of the functions $f_{2, k}$ satisfies the assumptions in either Lemma 2 or Lemma 3 (see Section 4). Appealing to these lemmas and using that $\tilde{f}_{\Lambda}^{(\Phi)}[f]$ is linear in $f$, we conclude:

1. singsupp $\tilde{f}_{\Lambda}^{(\Phi)}\left[f_{2}\right] \subset\left(S \cap V_{2}\right) \cup \Gamma_{\operatorname{supp} f_{2}}$,

2. the behavior of $\tilde{f}_{\Lambda}^{(\Phi)}\left[f_{2}\right]$ in a neighborhood of $x \in S \cap V_{2}, x \notin \Gamma$, is given by (2.9), (2.10);

3. the behavior of $\tilde{f}_{\Lambda}^{(\Phi)}\left[f_{2}\right]$ in a neighborhood of $x \in \Gamma_{\text {supp } f_{2}}, x \notin S$, is given by (2.11)-(2.13); and

4. the behavior of $\tilde{f}_{\Lambda}^{(\Phi)}\left[f_{2}\right]$ in a neighborhood of $x \in S \cap \Gamma_{\text {supp } f_{2}} \cap V_{2}$ is given by (2.14). 
Here it is understood that in formulas (2.9)-(2.14) one should replace $f$ by $f_{2}$.

Clearly, $f_{3} \in C_{0}^{\infty}\left(\mathbb{R}^{2}\right)$. By construction, there is no line $L$ which satisfies conditions (a)-(c) with $D=\operatorname{supp} f_{3}$. Similarly, using a partition of unity, represent $f_{3}$ in the form $f_{3}=\sum_{k} f_{3, k}$, where each of the functions $f_{3, k}$ satisfies the assumptions in Lemma 1 (see Section 3). Appealing to Lemma 1, we conclude:

5. singsupp $\tilde{f}_{\Lambda}^{(\Phi)}\left[f_{3}\right] \subset \Gamma_{\text {supp } f_{3}}$, and

6. the behavior of $\tilde{f}_{\Lambda}^{(\Phi)}\left[f_{3}\right]$ in a neighborhood of $x \in \Gamma_{\operatorname{supp} f_{3}}$ is given by (2.11)(2.13) with $f$ replaced by $f_{3}$.

Using Lemma 4 (see Section 4), we conclude:

7. singsupp $\tilde{f}_{\Lambda}^{(\Phi)}\left[f_{1}\right] \subset V_{1} \cup \Gamma_{\text {supp } f_{1}}$, and

8. the behavior of $\tilde{f}_{\Lambda}^{(\Phi)}\left[f_{1}\right]$ in a neighborhood of $x \in \Gamma_{\text {supp } f_{1}} \backslash V_{1}$ is given by (2.11)-(2.13) with $f$ replaced by $f_{1}$.

Since $V_{1} \rightarrow \bigcup_{j} L_{j}$ as $\epsilon \rightarrow 0, V_{2} \rightarrow S \backslash\left(\bigcup_{j} L_{j}\right)$ as $\epsilon, \delta \rightarrow 0$, and $\tilde{f}_{\Lambda}^{(\Phi)}[f]=$ $\tilde{f}_{\Lambda}^{(\Phi)}\left[f_{1}\right]+\tilde{f}_{\Lambda}^{(\Phi)}\left[f_{2}\right]+\tilde{f}_{\Lambda}^{(\Phi)}\left[f_{3}\right]$, combining items $1-8$ above proves Theorem 1.

Everywhere below we use the notation from Theorem 1. In particular, $D_{f}$ and $D_{\mu}$ denote values of the jumps of $f$ and $\mu$, respectively, and $n(x)$ denotes a unit vector perpendicular to a curve (either $S$ or $\Gamma$ ) where $x$ is located. Similarly, $R(x)$ denotes the radius of curvature of a curve (either $S$ or $\Gamma$ ) where $x$ is located. It is always assumed in what follows that the functions $f$ and $\mu$ satisfy not only the assumptions formulated in the corresponding lemmas, but also the assumptions in Theorem 1.

\section{LEMMA 1}

Lemma 1. Let the following assumptions hold.

1. $f \in C_{0}^{\infty}(D)$ and $\operatorname{diam} D$ is sufficiently small, so that

(1a) For any direction $\Theta \in S^{1}$ there is at most one line perpendicular to $\Theta$ and tangent to $\Gamma_{D}$;

(1b) $\operatorname{diam} D \ll \inf _{x \in \Gamma_{D}} R(x)$;

2. no line which intersects $D$ is tangent to $\Gamma$ at more than one point; and

3. the radius of curvature of $\Gamma$ is finite at any $x \in \Gamma_{D}$.

Then

$$
\text { singsupp } \tilde{f}_{\Lambda}^{(\Phi)} \subset \Gamma_{D}
$$

where $\Gamma_{D}$ is defined by (2.7). Fix any $x_{0} \in \Gamma_{D}$. Then

$$
\tilde{f}_{\Lambda}^{(\Phi)}(x)=-\frac{A\left(x_{0}, \mu, f\right)}{2 \pi} \operatorname{Im}\left\{\int_{0}^{\infty} \Psi_{\mu}(t, x) e^{i t h} d t\right\}, \quad x=x_{0}+h n\left(x_{0}\right),
$$

where $A\left(x_{0}, \mu, f\right)$ is defined by (2.12), and $\Psi_{\mu}(t, x) \in C^{\infty}\left([0, \infty) \times U_{\epsilon}\left(x_{0}\right)\right)$ for some $\epsilon>0$. Moreover, $\Psi_{\mu}$ admits the asymptotic expansion (2.13), which can be differentiated with respect to $t$ and $x$.

Proof. Let $\Omega \subset \frac{1}{2} S^{1}$ be a set such that for any $\Theta \in \Omega$ there is a point $y \in D$ with the following property: the line $L(\theta):=\left\{x(t)=y+t \Theta^{\perp}, t \in \mathbb{R}\right\}$ is tangent to $\Gamma$ (see Figure 3.1). Let $x_{0}(\theta)$ be the point of contact of the line $L(\theta)$ and $\Gamma$. According to Assumption (1a), the mapping $\theta \rightarrow x_{0}(\theta), \theta \in \Omega$, is one-to-one. Suppose, for example, that the half of the unit sphere $\frac{1}{2} S^{1}$ is chosen so that the directions $\Theta \in \Omega \subset \frac{1}{2} S^{1}$ point away from the center of curvature of $\Gamma$ at $x_{0}(\theta)$. Thus, $\Omega$ is 


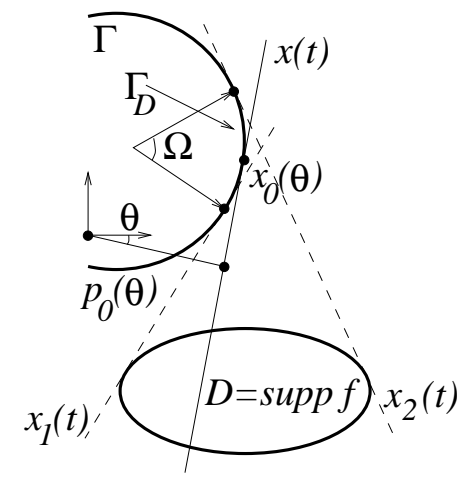

Figure 3.1. Illustration for Lemma 1.

the set of unit outward normals to $\Gamma_{D}$. Let $\epsilon>0$ be sufficiently small. Then $\Gamma$ splits $U_{\epsilon}\left(\Gamma_{D}\right)$ into two (possibly multiconnected) sets: $U_{\epsilon}^{+}\left(\Gamma_{D}\right)$ and $U_{\epsilon}^{-}\left(\Gamma_{D}\right)$. Let $U_{\epsilon}^{-}\left(\Gamma_{D}\right)$ be on the interior side of $\Gamma$ and $U_{\epsilon}^{+}\left(\Gamma_{D}\right)$ be on the exterior side of $\Gamma$; that is, the directions $\Theta \in \Omega$ point from $U_{\epsilon}^{-}\left(\Gamma_{D}\right)$ to $U_{\epsilon}^{+}\left(\Gamma_{D}\right)$. In view of (2.4), the first step is to find the asymptotics of the integral

$$
I(\sigma, \Theta)=\int_{\mathbb{R}^{2}} b(y, \theta) f(y) e^{i \sigma \Theta \cdot y} d y, \quad \sigma \rightarrow \infty,
$$

when $\Theta \in \Omega$. Denote $p_{0}(\theta)=x_{0}(\theta) \cdot \Theta$.

Using the assumptions in Theorem 1 , denote also

$$
\mu_{n s}(x):=\sum_{\substack{k \\ \partial D_{\mu, k} \cap \Gamma_{D} \neq \varnothing}} \mu_{k}(x) \chi_{\mu, k}(x), \quad \mu_{s m}:=\mu-\mu_{n s} .
$$

Clearly, $\mu=\mu_{s m}+\mu_{n s}$, and the functions $\mu_{n s}$ and $\mu_{s m}$ have the following properties:

1. $\mu_{n s} \in C^{\infty}\left(U_{\epsilon}\left(\Gamma_{D}\right) \backslash \Gamma\right)$ for some $\epsilon>0$;

2. $\mu_{n s} \neq 0$ in $U_{\epsilon}^{-}\left(\Gamma_{D}\right)$;

3. $\mu_{n s} \equiv 0$ in $U_{\epsilon}^{+}\left(\Gamma_{D}\right)$;

4. $\mu_{s m} \in C^{\infty}\left(U_{\epsilon}\left(\Gamma_{D}\right)\right)$; and

5. the lines $\left\{x(t)=y+t \Theta^{\perp}, t \in \mathbb{R}\right\}, y \in D, \Theta \in \Omega$, intersect the discontinuities of $\mu_{s m}$ transversely.

Fix any $\Theta \in \Omega$ and $p<p_{0}(\theta)$. Let $t=\eta(\theta, p)$ and $t=\nu(\theta, p)$ be the points of intersection of the line $\left\{p \Theta+t \Theta^{\perp}, t \in \mathbb{R}\right\}$ and $\Gamma$ in a neighborhood of $x_{0}(\theta)$ (that is, $\eta(\theta, p), \nu(\theta, p) \rightarrow x_{0}(\theta) \cdot \Theta^{\perp}$ as $\left.p \rightarrow p_{0}(\theta)\right)$. Assumptions 2 and 3 in Lemma 1 imply that if $p$ is sufficiently close to $p_{0}(\theta)$, then there are precisely two such points. Put $y=p \Theta+t \Theta^{\perp}$ in (3.3) and rewrite the integral in (3.3) as follows:

$$
I(\sigma, \Theta)=\int_{-\infty}^{\infty} G(\theta, p) e^{i \sigma p} d p
$$

where

$$
G(\theta, p)=\int_{-\infty}^{\infty} b\left(p \Theta+t \Theta^{\perp}, \theta\right) f\left(p \Theta+t \Theta^{\perp}\right) d t .
$$

By Assumption (1a) in Lemma 1 , any line $\left\{p \Theta+t \Theta^{\perp}, t \in \mathbb{R}\right\}, p \neq p_{0}(\theta)$, that intersects $D$ is transversal to $\Gamma$. This implies (see Proposition 1 in Section 8 ) that $G(\theta, \cdot) \in C^{\infty}\left(\mathbb{R} \backslash p_{0}(\theta)\right)$. Let us investigate the singularity of $G(\theta, p)$ as $p \rightarrow p_{0}(\theta)$. 
Substituting (2.5) into (3.5) and using the fact that $\mu_{n s} \equiv 0$ in $U_{\epsilon}^{+}\left(\Gamma_{D}\right)$, we find that for $p$ sufficiently close to $p_{0}(\theta), p \leq p_{0}(\theta)$ :

$$
\begin{aligned}
2 G(\theta, p)= & \int_{-\infty}^{\eta} \exp \left\{-\int_{\eta}^{\nu} \mu_{n s} d s-\int_{t}^{\infty} \mu_{s m} d s\right\} f(y) d t \\
& +\int_{-\infty}^{\eta} \exp \left\{-\int_{-\infty}^{t} \mu_{s m} d s\right\} f(y) d t \\
& +\int_{\eta}^{\nu} \exp \left\{-\int_{t}^{\nu} \mu_{n s} d s-\int_{t}^{\infty} \mu_{s m} d s\right\} f(y) d t \\
& +\int_{\eta}^{\nu} \exp \left\{-\int_{\eta}^{t} \mu_{n s} d s-\int_{-\infty}^{t} \mu_{s m} d s\right\} f(y) d t \\
& +\int_{\nu}^{\infty} \exp \left\{-\int_{t}^{\infty} \mu_{s m} d s\right\} f(y) d t \\
& +\int_{\nu}^{\infty} \exp \left\{-\int_{\eta}^{\nu} \mu_{n s} d s-\int_{-\infty}^{t} \mu_{s m} d s\right\} f(y) d t
\end{aligned}
$$

where we have dropped the arguments of $\eta$ and $\nu$ and used the convention $\int \mu d s=$ $\int \mu\left(p \Theta+s \Theta^{\perp}\right) d s$. Proposition 2 in Section 8 and Theorem 4.5.1 of [RK, p.106] (see also [RK, pp.112-114]) imply

$$
\begin{gathered}
(\eta(\theta, p)+\nu(\theta, p)) / 2=\psi_{1}(\theta, p), p \leq p_{0}(\theta), \psi_{1}(\theta, p) \in C^{\infty}\left(\Omega \times\left[p_{0}(\theta)-\epsilon, p_{0}(\theta)\right]\right), \\
\nu(\theta, p)-\eta(\theta, p)=\psi_{2}(\theta, p)\left(p-p_{0}(\theta)\right)_{-}^{0.5}, \psi_{2}(\theta, p) \in C^{\infty}\left(\Omega \times\left[p_{0}(\theta)-\epsilon, p_{0}(\theta)\right]\right),
\end{gathered}
$$

where $\left(p-p_{0}(\theta)\right)_{-}=\max \left(p_{0}(\theta)-p, 0\right)$. The notation

$$
\psi(\theta, p) \in C^{\infty}\left(\Omega \times\left[p_{0}(\theta)-\epsilon, p_{0}(\theta)\right]\right)
$$

means that $\psi$ is $C^{\infty}$ in a neighborhood of any $(\theta, p) \in \Omega \times\left[p_{0}(\theta)-\epsilon, p_{0}(\theta)\right]$, where $\epsilon>0$ is sufficiently small. Using (3.7), properties 4 and 5 formulated above (3.4), and Proposition 1 in Section 8, one can easily show that the integrals

$$
\begin{aligned}
& \int_{-\infty}^{(\eta+\nu) / 2} \exp \left\{-\int_{t}^{\infty} \mu_{s m} d s\right\} f(y) d t, \int_{-\infty}^{(\eta+\nu) / 2} \exp \left\{-\int_{-\infty}^{t} \mu_{s m} d s\right\} f(y) d t \\
& \int_{(\eta+\nu) / 2}^{\infty} \exp \left\{-\int_{t}^{\infty} \mu_{s m} d s\right\} f(y) d t, \int_{(\eta+\nu) / 2}^{\infty} \exp \left\{-\int_{-\infty}^{t} \mu_{s m} d s\right\} f(y) d t \\
& \quad \in C^{\infty}\left(\Omega \times\left[p_{0}(\theta)-\epsilon, p_{0}(\theta)\right]\right) .
\end{aligned}
$$

Changing variables $v=t-(\eta+\nu) / 2$, expanding $\mu_{n s}(x), x \in U_{\epsilon}^{-}\left(\Gamma_{D}\right)$, in the Taylor series in a neighborhood of $x=x_{0}(\theta)$, using the series representation for the exponentials containing $\mu_{n s}$, and taking into account (3.7), we prove that $G(\theta, p)$ admits an expansion in smoothness of the type $G(\theta, p) \sim \sum_{k \geq 0} g_{k}(\theta)\left[\left(p-p_{0}(\theta)\right)_{-}^{0.5}\right]^{k}, p \rightarrow$ $p_{0}(\theta)$, where $g_{k}(\theta) \in C^{\infty}(\Omega)$. This is equivalent to the existence of two functions $\varphi_{1,2}$ such that

$G(\theta, p)=\varphi_{1}(\theta, p)\left(p-p_{0}(\theta)\right)_{-}^{0.5}+\varphi_{2}(\theta, p), \varphi_{1,2}(\theta, p) \in C^{\infty}\left(\Omega \times\left[p_{0}(\theta)-\epsilon, p_{0}(\theta)\right]\right)$.

Let us find the leading singular term of $G(\theta, p)$ as $p \rightarrow p_{0}(\theta)$. Keeping only the highest order singular terms $\left(p-p_{0}(\theta)\right)_{-}^{0.5}$ and, in particular, dropping all the 
smooth terms, we find that

$$
\begin{aligned}
2 G(\theta, p) \sim & \left(1-\hat{\mu}_{n s}(\theta, p)\right) \int_{-\infty}^{\eta} \exp \left\{-\int_{t}^{\infty} \mu_{s m} d s\right\} f(y) d t \\
& +\int_{-\infty}^{\eta} \exp \left\{-\int_{-\infty}^{t} \mu_{s m} d s\right\} f(y) d t \\
& +\int_{\eta}^{\nu} \exp \left\{-\int_{t}^{\infty} \mu_{s m} d s\right\} f(y) d t \\
& +\int_{\eta}^{\nu} \exp \left\{-\int_{-\infty}^{t} \mu_{s m} d s\right\} f(y) d t \\
& +\int_{\nu}^{\infty} \exp \left\{-\int_{t}^{\infty} \mu_{s m} d s\right\} f(y) d t \\
& +\left(1-\hat{\mu}_{n s}(\theta, p)\right) \int_{\nu}^{\infty} \exp \left\{-\int_{-\infty}^{t} \mu_{s m} d s\right\} f(y) d t \\
\sim & -\hat{\mu}_{n s}(\theta, p)\left[\int_{-\infty}^{\eta} \exp \left\{-\int_{t}^{\infty} \mu_{s m} d s\right\} f(y) d t\right. \\
& \left.+\int_{\nu}^{\infty} \exp \left\{-\int_{-\infty}^{t} \mu_{s m} d s\right\} f(y) d t\right]
\end{aligned}
$$

where $\hat{\mu}_{n s}$ denotes the classical Radon transform of $\mu_{n s}$. Theorem 4.5.1 of [RK, p.106] implies

$$
\hat{\mu}_{n s}(\theta, p) \sim-2 \sqrt{2 R(\theta)} D_{\mu}(\theta)\left(p-p_{0}(\theta)\right)_{-}^{0.5}, \quad p \rightarrow p_{0}(\theta),
$$

where $R(\theta):=R\left(x_{0}(\theta)\right)$ is the radius of curvature of $\Gamma$ at $x_{0}(\theta)$, and $D_{\mu}(\theta)$ is the jump of $\mu(x)$ across $\Gamma$ at $x_{0}(\theta)$. Taking into account the formulas $\eta\left(\theta, p_{0}(\theta)\right)=$ $\nu\left(\theta, p_{0}(\theta)\right)=x_{0}(\theta) \cdot \Theta^{\perp}$ and

$$
\begin{gathered}
\int_{t}^{\infty} \mu d s=\int_{t}^{\infty} \mu_{s m} d s, \quad \int_{-\infty}^{t} \mu d s=\int_{-\infty}^{t} \mu_{s m} d s, \\
\text { if } p=p_{0}(\theta), p_{0}(\theta) \Theta+t \Theta^{\perp} \in D,
\end{gathered}
$$

and using (3.9), (3.10), we obtain an explicit expression for the leading singular term of $G(\theta, p)$ as $p \rightarrow p_{0}(\theta)$ :

$$
\begin{aligned}
G(\theta, p) \sim & A\left(x_{0}(\theta), \mu, f\right) \sqrt{2 R(\theta)} D_{\mu}(\theta)\left(p-p_{0}(\theta)\right)_{-}^{0.5}, \quad p \rightarrow p_{0}(\theta), \\
A\left(x_{0}(\theta), \mu, f\right)= & \int_{-\infty}^{0} \exp \left[-\int_{t}^{\infty} \mu\left(x_{0}(\theta)+s \Theta^{\perp}\right) d s\right] f\left(x_{0}(\theta)+t \Theta^{\perp}\right) d t \\
& +\int_{0}^{\infty} \exp \left[-\int_{-\infty}^{t} \mu\left(x_{0}(\theta)+s \Theta^{\perp}\right) d s\right] f\left(x_{0}(\theta)+t \Theta^{\perp}\right) d t .
\end{aligned}
$$

Using (3.4), (3.11), and well-known results on the asymptotics of the Fourier transform (see e.g. Lemma 14.5.5 in [RK, p.421], and [W, pp. 77-79]), we get

$$
I(\sigma, \Theta)=\Psi_{1}(\sigma, \theta) e^{i \sigma p_{0}(\theta)},
$$

where

$$
\Psi_{1}(\sigma, \theta) \sim \sum_{k \geq 0} \frac{c_{k}(\theta)}{\sigma^{k+1.5}}, \quad \sigma \rightarrow \infty
$$




$$
c_{0}(\theta)=-A\left(x_{0}(\theta), \mu, f\right) \sqrt{2 R(\theta)} D_{\mu}(\theta) \Gamma(1.5) e^{-i \frac{3 \pi}{4}},
$$

and $\Gamma(t)$ is the gamma function.

By assumption 3 in Lemma $1, R(\theta)<\infty, \theta \in \Omega$. Therefore Lemma 5.9.1 of [RK, p.181] implies that $p_{0}(\theta) \in C^{\infty}(\Omega)$. This together with equations (3.4) and (3.8) implies that $I(\sigma \Theta)$ is smooth when $\Theta \in \Omega$. Using (3.12), we conclude that the function $\Psi_{1}$ is smooth.

Clearly, boundary points of the set $\Omega \subset S^{1}$ (which can be multiconnected) correspond to the directions $\Theta_{m}$ such that the lines $\left\{x_{m}(t)=p_{0}\left(\theta_{m}\right) \Theta_{m}+t \Theta_{m}^{\perp}, t \in\right.$ $\mathbb{R}\}, m=1,2, \ldots$, are tangent to $\operatorname{supp} f$ (see Figure 3.1). Since the directions $\Theta \in \Omega$ are exterior normals to $\Gamma$ at the points $x_{0}(\theta)$, the definition of the boundary directions $\Theta_{m}$ can be written as follows. Fix any $y \in x_{m}(t)$. Then $\Theta_{m}$ is such that

$$
\Theta_{m} \in \Omega \text { and either }(x-y) \cdot \Theta_{m} \leq 0, \forall x \in D, \text { or }(x-y) \cdot \Theta_{m} \geq 0, \forall x \in D .
$$

Condition (3.15) implies that $f(x)$ vanishes with all derivatives as $x$ approaches the line $x_{m}(t)$. Therefore, (3.5) yields that $G\left(\theta_{m}, p\right)$ is $C^{\infty}$ in $p$ in a neighborhood of $p_{0}\left(\theta_{m}\right)$. This together with (3.8) implies that all derivatives of $\varphi_{1}\left(\theta_{m}, p\right)$ with respect to $p$ vanish at $p=p_{0}\left(\theta_{m}\right)$. In view of (3.4) and (3.8), the coefficients $c_{k}(\theta)$, used in (3.13), are finite linear combinations of derivatives of $\varphi_{1}(\theta, p)$ with respect to $p$ evaluated at $p=p_{0}(\theta)$. Thus, $c_{k} \in C_{0}^{\infty}(\Omega), k=0,1, \ldots$.

Let $\kappa$ be any function with the following properties: $\kappa(p) \in C_{0}^{\infty}([-\epsilon, \epsilon])$ and $\kappa(p) \equiv 1$ on $[-\epsilon / 2, \epsilon / 2]$. Substituting (3.8) into (3.4) and changing variables, we find that

$$
\begin{aligned}
I(\sigma, \Theta)= & \int_{\left|p-p_{0}(\theta)\right|>\epsilon / 2}\left(1-\kappa\left(p-p_{0}(\theta)\right)\right) G(\theta, p) e^{i \sigma p} d p \\
& +\int_{\left|p-p_{0}(\theta)\right|<\epsilon} \kappa\left(p-p_{0}(\theta)\right) G(\theta, p) e^{i \sigma p} d p \\
= & \int_{\left|p-p_{0}(\theta)\right|>\epsilon / 2}\left(1-\kappa\left(p-p_{0}(\theta)\right)\right) G(\theta, p) e^{i \sigma p} d p \\
& +e^{i \sigma p_{0}(\theta)} \int_{-\infty}^{\infty} \kappa(p)\left[\varphi_{1}\left(\theta, p+p_{0}(\theta)\right) p_{-}^{0.5}+\varphi_{2}\left(\theta, p+p_{0}(\theta)\right)\right] e^{i \sigma p} d p .
\end{aligned}
$$

Differentiating the last equation with respect to $\sigma$ and $\theta$, one verifies that the asymptotic expansion (3.13) can be differentiated with respect to $\sigma$ and $\theta$.

The final step is to obtain the asymptotics in smoothness of $\tilde{f}_{\Lambda}^{(\Phi)}(x)$. Consider the integral

$$
J_{\Omega}(\sigma, x):=\int_{\Omega} I(\sigma \Theta) e^{-i \sigma \Theta \cdot x} d \theta=\int_{\Omega} \Psi_{1}(\sigma, \theta) e^{i \sigma\left(p_{0}(\theta)-\Theta \cdot x\right)} d \theta .
$$

It is well-known that the stationary point of the phase $a(\theta, x)=p_{0}(\theta)-\Theta \cdot x$ corresponds to the direction $\Theta(x)$ such that the line $\{x+t \Theta(x), t \in \mathbb{R}\}$ is perpendicular to $\Gamma$ at the point of their intersection (see e.g. Lemma 5.9.2 in [RK, p.182]). Fix any $x_{0} \in \Gamma$ such that $n\left(x_{0}\right) \in \Omega$. Here $n\left(x_{0}\right)$ is the unit outward normal to $\Gamma$ at $x_{0}$. Take $x$ of the form $x=x_{0}+h n\left(x_{0}\right)$. Then, one can readily show that (see Lemma 5.9.2, [RK, p.182])

$$
a(\theta(x), x)=-h,\left.\quad \frac{\partial^{2} a(\theta, x)}{\partial \theta^{2}}\right|_{\theta=\theta(x)}=R\left(x_{0}\right)+h,
$$


where $R\left(x_{0}\right)$ is the radius of curvature of $\Gamma$ at $x_{0}$. As it was established above, $c_{k} \in C_{0}^{\infty}(\Omega), k=0,1, \ldots$ Substituting (3.13) into (3.16), using the fact that the stationary point of the phase $a(\theta, x)$ corresponds to the direction $\Theta=n\left(x_{0}\right)$, and applying the stationary phase method term by term, we get

$$
\begin{gathered}
J_{\Omega}(\sigma, x)=\Psi_{2}(\sigma, x) e^{-i \sigma h}, \quad x=x_{0}+h n\left(x_{0}\right), n\left(x_{0}\right) \in \Omega, \\
\Psi_{2}(\sigma, x) \sim \sum_{k \geq 0} \frac{d_{k}(x)}{\sigma^{k+2}}, \quad \sigma \rightarrow \infty \\
d_{0}(x)=c_{0}\left(n\left(x_{0}\right)\right) \sqrt{\frac{2 \pi}{R\left(x_{0}\right)+h}} e^{i \frac{\pi}{4}} .
\end{gathered}
$$

Using (3.14), we can rewrite the last equation as follows:

$$
d_{0}(x)=-\pi i A\left(x_{0}, \mu, f\right) D_{\mu}\left(x_{0}\right) \sqrt{\frac{R\left(x_{0}\right)}{R\left(x_{0}\right)+h}},
$$

where $A\left(x_{0}, \mu, f\right)$ is defined by $(2.12)$, and $D_{\mu}\left(x_{0}\right)$ is the value of the jump of $\mu$ at $x_{0}$. From the first equality in (3.16) it is obvious that $J_{\Omega}(\sigma, x) \in C^{\infty}\left([0, \infty) \times \mathbb{R}^{2}\right)$. Let $V$ be an open set containing $\Gamma_{D}$ such that given any $x \in V$ we have $x=x_{0}+h n\left(x_{0}\right)$ for some $x_{0} \in \Gamma_{D}$ and $h \in \mathbb{R}$, this representation is unique, and the function $h=h(x)$ is smooth. Clearly, such a set exists and $\operatorname{diam} V$ depends only on the geometry of $\Gamma_{D}$. In particular, diam $V$ depends on $\inf _{x \in \Gamma_{D}} R(x)$. Equation (3.18) implies that $\Psi_{2}(\sigma, x) \in C^{\infty}([0, \infty) \times V)$. From (3.16)-(3.18) we obtain

$$
\Psi_{2}(\sigma, x)=\int_{\Omega} \Psi_{1}(\sigma, \theta) e^{i \sigma(a(\theta, x)-a(\theta(x), x))} d \theta .
$$

Differentiating the last equation with respect to $\sigma$ and $x$, applying the stationary phase method, and taking into account that

$$
a(\theta, x)-\left.a(\theta(x), x)\right|_{\theta=\theta(x)}=\left.\frac{\partial}{\partial \theta}[a(\theta, x)-a(\theta(x), x)]\right|_{\theta=\theta(x)}=0,
$$

we verify that the derivatives of $\Psi_{2}$ with respect to $\sigma$ and $x$ can be obtained by differentiating the asymptotic expansion (3.18).

Since the set $\frac{1}{2} S^{1}$ in (2.6) can be chosen arbitrarily, we may suppose that $\Omega \subset$ $\frac{1}{2} S^{1}$. Using (3.18), (3.19), and $\left(3.20^{\prime}\right)$ in $(2.6)$, we find that

$$
\begin{aligned}
g_{\Omega}(x): & =\frac{2}{(2 \pi)^{2}} \operatorname{Re}\left\{\int_{0}^{\infty} \int_{\Omega} \int_{\mathbb{R}^{2}} b(y, \theta) f(y) e^{-i \sigma \Theta \cdot(x-y)} d y d \theta \sigma^{2} d \sigma\right\} \\
& =\frac{2}{(2 \pi)^{2}} \operatorname{Re}\left\{\int_{0}^{\infty} J_{\Omega}(\sigma, x) \sigma^{2} d \sigma\right\} \\
& =-\frac{A\left(x_{0}, \mu, f\right)}{2 \pi} \sqrt{\frac{R\left(x_{0}\right)}{R\left(x_{0}\right)+h}} \operatorname{Im}\left\{\int_{0}^{\infty} \Psi_{\mu}(\sigma, x) e^{i \sigma h} d \sigma\right\}, \\
x & =x_{0}+h n\left(x_{0}\right), n\left(x_{0}\right) \in \Omega, x \in V,
\end{aligned}
$$

where the function $\Psi_{\mu}$ has the following asymptotic expansion:

$$
\Psi_{\mu}(\sigma, x) \sim D_{\mu}\left(x_{0}\right)+\sum_{k \geq 1} \frac{d_{k}(x)}{\sigma^{k}}, \quad \sigma \rightarrow \infty .
$$


Here we have assumed implicitly that $A\left(x_{0}, \mu, f\right) \neq 0$ for $x_{0} \in \Gamma_{D}$. The coefficients $d_{k}$ in (3.22) differ from the coefficients $d_{k}$ in (3.19) by a constant factor. Since the function $\sqrt{R\left(x_{0}\right) /\left(R\left(x_{0}\right)+h\right)}$ is smooth near $h=0$ and equals 1 at $h=0$, we can absorb this function by the integral on the right-hand side of (3.21) without changing the first term in (3.22).

From (3.21) it follows that $x=x_{0}+h n\left(x_{0}\right) \notin \operatorname{singsupp} g_{\Omega}$ if $x \in V$ and $h \neq 0$. Recall that the set $V$ was defined in the paragraph following $\left(3.20^{\prime}\right)$. Using equation (22) of [GS, p.360], we see that the integral

$$
\int_{0}^{\infty} \sigma^{2} e^{-i \sigma \Theta \cdot(x-y)} d \sigma=2 i^{3}[\Theta \cdot(y-x)]^{-3}+(-i)^{2} \pi \delta^{\prime \prime}(\Theta \cdot(y-x)),
$$

which is obtained from the definition of $g_{\Omega}$ by retaining the integration with respect to $\sigma$, is a $C^{\infty}$ function of $\theta, x$, and $y$ when $\Theta \cdot(y-x) \neq 0$. By Assumption (1b) in Lemma $1, \Theta \cdot(y-x) \neq 0$ when $x \notin V, y \in D$, and $\Theta \in \Omega$. Therefore, $x=x_{0}+h n\left(x_{0}\right) \notin \operatorname{singsupp} g_{\Omega}$ if $x \notin V$ and $n\left(x_{0}\right) \in \Omega$.

Suppose now that $x_{0} \in \Gamma$ is chosen so that $n\left(x_{0}\right) \notin \Omega$. Integrating by parts in (3.16) and using the fact that derivative of the phase $a(\theta, x)=p_{0}(\theta)-\Theta \cdot x$ with respect to $\theta$ does not vanish when $\Theta \in \Omega$, we see that $J_{\Omega}(\sigma, x)=O\left(\sigma^{-\infty}\right), \sigma \rightarrow \infty$. Therefore, $x \notin \operatorname{singsupp} g_{\Omega}$ if $x=x_{0}+h n\left(x_{0}\right)$ and $n\left(x_{0}\right) \notin \Omega$.

Recall that $\Omega$ was chosen so that for any $\Theta \in \Omega$ there exists a line which is perpendicular to $\Theta$, tangent to $\Gamma$, and which intersects $D$. We have just established that singsupp $g_{\Omega} \subset \Gamma_{D}$ (cf. (3.1)). In view of (2.6), we have $\tilde{f}_{\Lambda}^{(\Phi)}=g_{\Omega}+g_{\Omega^{\prime}}$, where $\Omega^{\prime}=\frac{1}{2} S^{1} \backslash \Omega$. Fix any $\Theta \in \Omega^{\prime}$. The lines $\left\{x(t)=y+t \Theta^{\perp}, t \in \mathbb{R}\right\}, y \in D$, are transversal to $\Gamma$. Using Proposition 1 in Section 8, one proves that $G(\theta, p) \in$ $C_{0}^{\infty}\left(D_{\Theta}\right)$ in the $p$ variable, where $D_{\Theta}$ is the projection of $D=\operatorname{supp} f$ onto direction $\Theta$. Integrating by parts in (3.4), we find that $I(\sigma \Theta)=O\left(\sigma^{-\infty}\right), \sigma \rightarrow \infty$; and, therefore, the function $g_{\Omega^{\prime}}$ is $C^{\infty}$ smooth. This argument together with (3.21) and (3.22) proves Lemma 1.

\section{Lemmas $2-4$}

Denote $S=\operatorname{singsupp} f$, and let $\Omega_{\Gamma_{D}}$ and $\Omega_{S}$ be the sets of unit outward normals to $\Gamma_{D}$ and $S$, respectively, $\Omega_{\Gamma_{D}}, \Omega_{S} \subset S^{1}$. One has

Lemma 2. Let the following assumptions hold.

1. $f$ is piecewise smooth and $D=\operatorname{supp} f$ is sufficiently small, so that

(1a) $\Omega_{\Gamma_{D}} \cap \Omega_{S}=\varnothing$ (see Figure 4.1);

(1b) for any direction $\Theta \in \Omega_{\Gamma_{D}}$ there is only one line perpendicular to $\Theta$ and tangent to $\Gamma_{D}$;

(1c) $\operatorname{diam} D \ll \inf _{x \in \Gamma_{D}} R(x)$;

2. $S \cap \Gamma=\varnothing$;

3. no line which intersects $D$ is tangent to $S \cup \Gamma$ at more than one point; and

4. the radius of curvature of $\Gamma$ is finite at any $x \in \Gamma_{D}$.

Then

$$
\operatorname{singsupp} \tilde{f}_{\Lambda}^{(\Phi)} \subset S \cup \Gamma_{D} .
$$

In a neighborhood of $\Gamma_{D}$, the asymptotics in smoothness of $\tilde{f}_{\Lambda}^{(\Phi)}$ is given by (2.11)(2.13). In a neighborhood of $S$, the asymptotics in smoothness of $\tilde{f}_{\Lambda}^{(\Phi)}$ is given by (2.9), (2.10). 




FiguRE 4.1. Illustration for Lemma 2.

Proof. Since $\Omega_{\Gamma_{D}}$ and $\Omega_{S}$ are compact disjoint sets, there exists an even function $\chi \in C^{\infty}\left(S^{1}\right)$ such that $\chi \equiv 1$ on $\Omega_{\Gamma_{D}}$ and $\chi \equiv 0$ on $\Omega_{S}$. Using (2.6), define two functions:

$$
\begin{aligned}
& g_{1}(x)=\frac{2}{(2 \pi)^{2}} \operatorname{Re}\left(\int_{0}^{\infty} \int_{\frac{1}{2} S^{1} \backslash \Omega_{S}} \int_{\mathbb{R}^{2}} \chi(\theta) b(y, \theta) f(y) e^{-i \sigma \Theta \cdot(x-y)} d y d \theta \sigma^{2} d \sigma\right), \\
& g_{2}(x)=\frac{2}{(2 \pi)^{2}} \operatorname{Re}\left(\int_{0}^{\infty} \int_{\frac{1}{2} S^{1} \backslash \Omega_{\Gamma_{D}}} \int_{\mathbb{R}^{2}}(1-\chi(\theta)) b(y, \theta) f(y) e^{-i \sigma \Theta \cdot(x-y)} d y d \theta \sigma^{2} d \sigma\right) .
\end{aligned}
$$

The proof of Lemma 1 carries over to the analysis of the function $g_{1}$. By assumption (1a), the lines $\left\{x(t)=y+t \Theta^{\perp}, t \in \mathbb{R}\right\}$, where $y \in D, \Theta \notin \Omega_{S}$, are transversal to $S$. Therefore, as in the proof of Lemma 1 , integration with respect to $\theta$ in the definition of $g_{1}$ can be confined to the set $\Omega_{\Gamma_{D}}$. Furthermore, analyzing (3.6), using the fact that any line $\left\{x(t)=p \Theta+t \Theta^{\perp}, t \in \mathbb{R}\right\}$ such that $x(t) \cap D \neq \varnothing$ and $\Theta \in \Omega_{\Gamma_{D}}$ intersects $S$ transversely, taking into account assumption (1b), appealing to Proposition 1 in Section 8, and noting that the points of intersection of the line $x(t)$ and $S$ are located outside the interval

$$
\left\{x \in \mathbb{R}^{2}: x=p \Theta+t \Theta^{\perp}, t \in[\eta(\theta, p), \nu(\theta, p)]\right\}, p \in\left[p_{0}(\theta)-\epsilon, p_{0}(\theta)\right],
$$

(see Figure 4.1), we conclude that $G(\theta, \cdot) \in C^{\infty}\left(\mathbb{R} \backslash p_{0}(\theta)\right), \Theta \in \Omega_{\Gamma_{D}}$, and properties (3.8) and (3.11) still hold. The rest of the argument goes without changes, and we establish that singsupp $g_{1} \subset \Gamma_{D}$ and the asymptotics in smoothness of $g_{1}$ in a neighborhood of $\Gamma_{D}$ is given by (2.11)-(2.13).

Furthermore, the lines $\left\{x(t)=y+t \Theta^{\perp}, t \in \mathbb{R}\right\}, y \in D, \Theta \notin \Omega_{\Gamma_{D}}$, are transversal to $\Gamma$. Therefore, $(1-\chi(\theta)) b(y, \theta) \in C^{\infty}\left(D \times S^{1}\right)$, and we can apply Theorem 5.9.2 of [RK, p.188] (see also [K1] and Proposition 3 in Section 8), which proves that singsupp $g_{2} \subset S$ and the asymptotics in smoothness of $g_{2}$ in a neighborhood of $S$ is given by (2.9), (2.10).

Since $\tilde{f}_{\Lambda}^{(\Phi)}=g_{1}+g_{2}$, this finishes the proof of Lemma 2 .

Lemma 3. Let the following assumptions hold. 


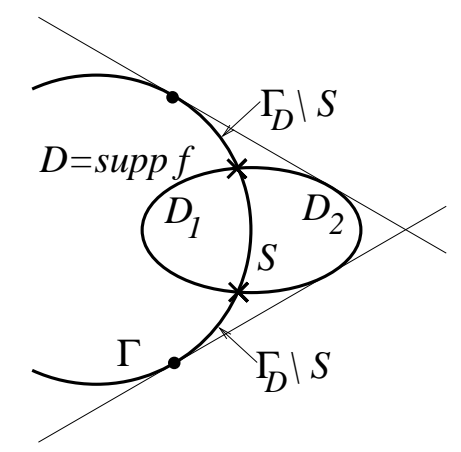

FiguRE 4.2. Illustration for Lemma 3.

1. $f$ can be represented in the form

$$
f(x)=\psi_{1}(x) \chi_{D_{1}}(x)+\psi_{2}(x) \chi_{D_{2}}(x), \psi_{1}, \psi_{2} \in C_{0}^{\infty}(D),
$$

where $D=D_{1} \cup D_{2}$ is the union of two open connected sets $D_{1}, D_{2} \subset \mathbb{R}^{2}$;

2. the boundary $S=\operatorname{singsupp} f$ between $D_{1}$ and $D_{2}$ is a connected smooth curve that has nonvanishing curvature at every point $x \in S$;

3. $D$ is sufficiently small:

(3a) for any direction $\Theta \in S^{1}$ there is at most one line perpendicular to $\Theta$ and tangent to $\Gamma_{D}$

(3b) no line tangent to $S$ intersects the set (either $D_{1}$ or $D_{2}$ ) that lies on the interior side of $S$;

(3c) $\operatorname{diam} D \ll \inf _{x \in \Gamma_{D}} R(x)$;

4. $\mu$ is discontinuous across $S$, that is $S \subset \Gamma_{D}$;

5. no line which intersects $D$ is tangent to $\Gamma$ at more than one point; and

6. the radius of curvature of $\Gamma$ is finite at any $x \in \Gamma_{D}$.

Then

$$
\operatorname{singsupp} \tilde{f}_{\Lambda}^{(\Phi)} \subset \Gamma_{D}
$$

In a neighborhood of $\Gamma_{D} \backslash S$, the asymptotics in smoothness of $\tilde{f}_{\Lambda}^{(\Phi)}$ is given by (2.11)-(2.13). In a neighborhood of $S$, the asymptotics in smoothness of $\tilde{f}_{\Lambda}^{(\Phi)}$ is given by (2.14).

Proof. Let $D_{1}$ be on the interior side of $S$, and $D_{2}$ be on the exterior side of $S$ (see Figure 4.2). Using (4.2), represent $f$ as follows:

$$
f(x)=\left(\psi_{1}(x)-\psi_{2}(x)\right) \chi_{D_{1}}(x)+\psi_{2}(x) .
$$

Assumptions 3, 5, and 6, imply that $\psi_{2}$ satisfies the assumptions in Lemma 1 . Therefore, Lemma 1 describes the singularities of $\tilde{f}_{\Lambda}^{(\Phi)}[f]$ in the case $f=\psi_{2} \in$ $C_{0}^{\infty}(D)$. Since $\tilde{f}_{\Lambda}^{(\Phi)}[f]$ depends linearly on $f$, it remains to analyze the function $\tilde{f}_{\Lambda}^{(\Phi)}\left[f_{n s}\right]$, where $f_{n s}:=\left(\psi_{1}-\psi_{2}\right) \chi_{D_{1}}$. 
Define two functions

$$
\begin{aligned}
& g_{1}(x)=\frac{2}{(2 \pi)^{2}} \operatorname{Re}\left(\int_{0}^{\infty} \int_{\Omega_{S}} \int_{\mathbb{R}^{2}} b(y, \theta) f_{n s}(y) e^{-i \sigma \Theta \cdot(x-y)} d y d \theta \sigma^{2} d \sigma\right), \\
& g_{2}(x)=\frac{2}{(2 \pi)^{2}} \operatorname{Re}\left(\int_{0}^{\infty} \int_{\frac{1}{2} S^{1} \backslash \Omega_{S}} \int_{\mathbb{R}^{2}} b(y, \theta) f_{n s}(y) e^{-i \sigma \Theta \cdot(x-y)} d y d \theta \sigma^{2} d \sigma\right),
\end{aligned}
$$

where $\Omega_{S}$ is the set of unit outward normals to $S$. Similarly to (3.4), (3.5), define

$$
I(\sigma \Theta)=\int_{-\infty}^{\infty} G(\theta, p) e^{i \sigma p} d p, G(\theta, p)=\int_{-\infty}^{\infty} b\left(p \Theta+t \Theta^{\perp}, \theta\right) f_{n s}\left(p \Theta+t \Theta^{\perp}\right) d t .
$$

First, investigate singularities of $g_{1}$. Clearly, $G(\theta, \cdot) \in C^{\infty}\left(\mathbb{R} \backslash p_{0}(\theta)\right)$ for any $\theta \in$ $\Omega_{\Gamma_{D}}$. By construction, $f_{n s} \equiv 0$ in $D_{2}$. Using assumption (3b), the analog of (3.6) becomes

$$
\begin{aligned}
2 G(\theta, p)= & \int_{\eta}^{\nu} \exp \left\{-\int_{t}^{\nu} \mu_{n s} d s-\int_{t}^{\infty} \mu_{s m} d s\right\} f_{n s}(y) d t \\
& +\int_{\eta}^{\nu} \exp \left\{-\int_{\eta}^{t} \mu_{n s} d s-\int_{-\infty}^{t} \mu_{s m} d s\right\} f_{n s}(y) d t \\
& p \in\left[p_{0}(\theta)-\epsilon, p_{0}(\theta)\right], \Theta \in \Omega_{S} .
\end{aligned}
$$

By assumption (3a), the mapping $\theta \rightarrow x_{0}(\theta), \theta \in \Omega_{S}$, is one-to-one. Expanding $\mu_{n s}(x)$ and $f_{n s}(x), x \in D_{1}$, in the Taylor series in a neighborhood of $x_{0}(\theta), \theta \in \Omega_{S}$, we see that equation (3.8) holds with some function $\varphi_{1} \in C^{\infty}\left(\Omega_{S} \times\left[p_{0}(\theta)-\epsilon, p_{0}(\theta)\right]\right)$ and $\varphi_{2} \equiv 0$. Similarly to (3.9)-(3.11), we find the leading singular term of $G(\theta, p)$ as $p \rightarrow p_{0}(\theta), \theta \in \Omega_{S}$ :

$$
\begin{gathered}
G(\theta, p) \sim \frac{1}{2}\left[\int_{\eta}^{\nu} \exp \left\{-\int_{t}^{\infty} \mu_{s m} d s\right\} f_{n s}(y) d t\right. \\
\left.\quad+\int_{\eta}^{\nu} \exp \left\{-\int_{-\infty}^{t} \mu_{s m} d s\right\} f_{n s}(y) d t\right] \\
\sim-2 \sqrt{2 R(\theta)} D_{f}(\theta)\left(p-p_{0}(\theta)\right)_{-}^{0.5} \\
\quad \times \frac{1}{2}\left[\exp \left\{-\int_{0}^{\infty} \mu\left(x_{0}(\theta)+s \Theta^{\perp}\right) d s\right\}\right. \\
\left.\quad+\exp \left\{-\int_{-\infty}^{0} \mu\left(x_{0}(\theta)+s \Theta^{\perp}\right) d s\right\}\right] \\
=-2 \sqrt{2 R(\theta)} D_{f}(\theta) b\left(x_{0}(\theta), \theta\right)\left(p-p_{0}(\theta)\right)_{-}^{0.5}, \quad p \rightarrow p_{0}(\theta),
\end{gathered}
$$

where we have used the equality $\lim _{x \rightarrow x_{0}}(\theta), x \in D_{1} f_{n s}(x)=-D_{f}(\theta)$. Therefore, the analog of equations (3.12)-(3.14) becomes

$$
\begin{aligned}
I(\sigma \Theta) & =\Psi_{1}(\sigma, \theta) e^{i \sigma p_{0}(\theta)}, \Psi_{1}(\sigma, \theta) \sim \sum_{k \geq 0} \frac{c_{k}(\theta)}{\sigma^{k+1.5}}, \quad \sigma \rightarrow \infty, \\
c_{0}(\theta) & =2 \sqrt{2 R(\theta)} D_{f}(\theta) b\left(x_{0}(\theta), \theta\right) \Gamma(1.5) e^{-i \frac{3 \pi}{4}} .
\end{aligned}
$$

Let $y$ be a boundary point of $S$, that is, $y \in S \cap \partial D$, and let $n(y)$ be the corresponding unit outward normal. Clearly, $n(y)$ is a boundary direction of the set $\Omega_{S}$. Since $\left|f_{n s}(x)\right| \leq c_{m}|x-y|^{m}$ as $x \rightarrow y$ for all $m \geq 0$ and some constants $c_{m}>0$, this implies that all derivatives of $\varphi_{1}(\theta, p)$ with respect to $p$ evaluated at $p=p_{0}(\theta)$ 
and $\Theta=n(y)$ vanish. Since the coefficients $c_{k}(\theta)$, used in (4.8), are finite linear combinations of derivatives of $\varphi_{1}(\theta, p)$ with respect to $p$ evaluated at $p=p_{0}(\theta)$, we conclude that $c_{k} \in C_{0}^{\infty}\left(\Omega_{S}\right), k=0,1, \ldots$

The rest of the argument from the proof of Lemma 1 can be adapted with almost no changes. Basically, one has to replace $-A\left(x_{0}, \mu, f\right)$ by $2 b\left(x_{0}, n\left(x_{0}\right)\right)$ and $D_{f}$ by $D_{\mu}$ in equations $\left(3.20^{\prime}\right)-(3.22)$. This yields that $\operatorname{singsupp} g_{1}=S$, and the asymptotics in smoothness of $g_{1}$ in a neighborhood of $S$ is given by (2.9), (2.10).

Consider now the function $g_{2}$ defined in (4.5). Since $D_{1}=\operatorname{supp} f_{n s}$, we have to integrate only over the cone with the opening $\Omega_{\Gamma_{D_{1} \backslash S}}$, which consists of the unit outward normals to $\Gamma_{D_{1}} \backslash S$. Formulas (3.12)-(3.14) still hold provided $\Theta \in \Omega_{\Gamma_{D_{1}} \backslash S}$. The rest of the argument from the proof of Lemma 1 can be used with almost no changes. Basically, one has to replace $f$ and $\Omega$ by $f_{n s}$ and $\Omega_{\Gamma_{D_{1} \backslash S}}$, respectively, in equations (3.15)-(3.22). This yields that singsupp $g_{2} \subset \Gamma_{D_{1}} \backslash S$, and the asymptotics in smoothness of $g_{2}$ in a neighborhood of $\Gamma_{D_{1}} \backslash S$ is given by (2.11)-(2.13) (with $f$ replaced by $\left.f_{n s}\right)$.

Since $f=\psi_{2}+f_{n s}, f=\psi_{2}$ on any line tangent to $S$, the singularities of the local tomography function corresponding to $\psi_{2}$ are described by Lemma 1 (with $f$ replaced by $\psi_{2}$ ), and $g_{1}+g_{2}$ is the local tomography function corresponding to $f_{n s}$, we finish the proof of Lemma 3 .

Lemma 4. Denote $D=\operatorname{supp} f$ and suppose $f$ satisfies the assumptions in Theorem 1. Suppose also that there exist lines $L_{j}, j \in J$, such that

(a) $L_{j} \cap D \neq \varnothing$;

(b) $L_{j}$ is tangent to $\Gamma$;

and at least one of the following conditions holds:

(c1) $L_{j}$ is tangent to $S \cup \Gamma$ at more than one point, or

(c2) the radius of curvature of $\Gamma$ at $\Gamma \cap L_{j}$ equals infinity.

Let $\Theta_{j}$ be a unit vector perpendicular to $L_{j}$. Then

$$
\operatorname{singsupp} \tilde{f}_{\Lambda}^{(\Phi)} \subset \Gamma_{D} \cup D^{*},
$$

where

$$
D^{*}:=\left\{x \in \mathbb{R}^{2}:(x-y) \cdot \Theta_{j}=0 \text { for some } j \in J \text { and } y \in D\right\} .
$$

Moreover, if $\Gamma_{D} \backslash D^{*} \neq \varnothing$, then the behavior of $\tilde{f}_{\Lambda}^{(\Phi)}$ in a neighborhood of any $x_{0} \in \Gamma_{D} \backslash D^{*}$ is given by (2.11)-(2.13).

Proof. Let $\Omega_{2 \epsilon} \subset \frac{1}{2} S^{1}$ be a $2 \epsilon$-neighborhood of the set $\bigcup_{j} \Theta_{j}$. Fix a real-valued function $\chi_{\epsilon} \in C_{0}^{\infty}\left(\Omega_{2 \epsilon}\right)$ such that $\chi_{\epsilon} \equiv 1$ on $\Omega_{\epsilon}$. Define two functions

$$
\begin{aligned}
& g_{1}(x)=\frac{2}{(2 \pi)^{2}} \operatorname{Re}\left(\int_{0}^{\infty} \int_{\frac{1}{2} S^{1} \backslash \Omega_{\epsilon}} \int_{\mathbb{R}^{2}}\left(1-\chi_{\epsilon}(\theta)\right) b(y, \theta) f(y) e^{-i \sigma \Theta \cdot(x-y)} d y d \theta \sigma^{2} d \sigma\right), \\
& g_{2}(x)=\frac{2}{(2 \pi)^{2}} \operatorname{Re}\left(\int_{0}^{\infty} \int_{\Omega_{2 \epsilon}} \int_{\mathbb{R}^{2}} \chi_{\epsilon}(\theta) b(y, \theta) f(y) e^{-i \sigma \Theta \cdot(x-y)} d y d \theta \sigma^{2} d \sigma\right) .
\end{aligned}
$$

Let us find the singularities of $g_{1}$. Using a partition of unity, represent $f$ as follows: $f=\sum_{k} f_{k}$. Denote $D_{k}=\operatorname{supp} f_{k}$ and $\Omega_{k}=\Omega_{\Gamma_{\text {supp } f_{k}}}$. The functions $f_{k}$ are chosen so that they satisfy the following conditions: 
(a) No line that intersects $D_{k}$ and is perpendicular to $\Theta \notin \Omega_{\epsilon}$ is tangent to $\Gamma \cup \operatorname{singsupp} f_{k}$ at more than one point.

(b) The radius of curvature of $\Gamma$ is finite at any $x \in \Gamma_{D_{k}}$ such that $n(x) \notin \Omega_{\epsilon}$.

(c) $\operatorname{diam} D_{k} \ll \inf _{x \in \Gamma_{D_{k}}} R(x)$.

Following the proof of Lemma 1,2, or 3, we can describe the singularities of $\tilde{f}_{\Lambda}^{(\Phi)}\left[f_{k}\right]$. Suppose, for example, that $f_{k} \in C_{0}^{\infty}\left(\mathbb{R}^{2}\right)$. Clearly, equations (3.12)(3.14) still hold for $\Theta \in \Omega_{k} \backslash \Omega_{\epsilon}$ with $f$ replaced by $f_{k}$. Instead of (3.16), we have

$$
\begin{aligned}
J_{\Omega_{k}}(\sigma, x) & :=\int_{\Omega_{k}}\left(1-\chi_{\epsilon}(\theta)\right) I(\sigma \Theta) e^{-i \sigma \Theta \cdot x} d \theta \\
& =\int_{\Omega_{k}}\left(1-\chi_{\epsilon}(\theta)\right) \Psi_{1}(\sigma, \theta) e^{i \sigma\left(p_{0}(\theta)-\Theta \cdot x\right)} d \theta .
\end{aligned}
$$

Equations (3.18) and (3.19) still hold provided $n\left(x_{0}\right) \in \Omega_{k}$, and the analog of $\left(3.20^{\prime}\right)$ becomes

$$
d_{0}(x)=-\pi i\left[1-\chi_{\epsilon}\left(n\left(x_{0}\right)\right)\right] A\left(x_{0}, \mu, f_{k}\right) D_{\mu}\left(x_{0}\right) \sqrt{\frac{R\left(x_{0}\right)}{R\left(x_{0}\right)+h}} .
$$

Clearly, $d_{0}(x)=0, x=x_{0}+h n\left(x_{0}\right)$, if $n\left(x_{0}\right) \in \Omega_{\epsilon}$. Following the rest of the proof of Lemma 1, we see that

$$
\operatorname{singsupp} \tilde{f}_{\Lambda}^{(\Phi)}\left[f_{k}\right] \subset\left\{x \in \Gamma_{D_{k}}: n(x) \notin \Omega_{\epsilon}\right\},
$$

and the behavior of $\tilde{f}_{\Lambda}^{(\Phi)}\left[f_{k}\right]$ in a neighborhood of singsupp $\tilde{f}_{\Lambda}^{(\Phi)}\left[f_{k}\right]$ is described by (2.11)-(2.13) with $f$ and $D_{\mu}\left(x_{0}\right)$ replaced by $f_{k}$ and $\left[1-\chi_{\epsilon}\left(n\left(x_{0}\right)\right)\right] D_{\mu}\left(x_{0}\right)$, respectively.

In a similar fashion, following the proofs of Lemmas 2 and 3, we find that in all three cases

$$
\operatorname{singsupp} \tilde{f}_{\Lambda}^{(\Phi)}\left[f_{k}\right] \subset\left\{x \in \Gamma_{D_{k}} \cup \operatorname{singsupp} f_{k}: n(x) \notin \Omega_{\epsilon}\right\},
$$

and the behavior of $\tilde{f}_{\Lambda}^{(\Phi)}\left[f_{k}\right]$ in a neighborhood of its singular support is described by (2.9)-(2.14) with $f, D_{f}\left(x_{0}\right)$, and $D_{\mu}\left(x_{0}\right)$ replaced by $f_{k},\left[1-\chi_{\epsilon}\left(n\left(x_{0}\right)\right)\right] D_{f_{k}}\left(x_{0}\right)$, and $\left[1-\chi_{\epsilon}\left(n\left(x_{0}\right)\right)\right] D_{\mu}\left(x_{0}\right)$, respectively. Therefore,

$$
\text { singsupp } g_{1} \subset\left\{x \in \Gamma_{D} \cup \operatorname{singsupp} f: n(x) \notin \Omega_{\epsilon}\right\} \text {, }
$$

and the behavior of $g_{1}$ in a neighborhood of its singular support is described by (2.9)-(2.14) with $D_{f}\left(x_{0}\right)$ and $D_{\mu}\left(x_{0}\right)$ replaced by $\left[1-\chi_{\epsilon}\left(n\left(x_{0}\right)\right)\right] D_{f}\left(x_{0}\right)$ and $\left[1-\chi_{\epsilon}\left(n\left(x_{0}\right)\right)\right] D_{\mu}\left(x_{0}\right)$, respectively.

Consider now the function $g_{2}$. If $x$ is chosen so that $|\Theta \cdot(x-y)| \geq \gamma>0$ for all $\Theta \in \Omega_{2 \epsilon}$ and $y \in D$, then equation (3.23) implies that $x \notin \operatorname{singsupp} g_{2}$. Since $\gamma>0$ can be chosen arbitrarily small, we conclude that

$$
\text { singsupp } g_{2} \subset\left\{x \in \mathbb{R}^{2}:(x-y) \cdot \Theta=0 \text { for some } \Theta \in \Omega_{2 \epsilon} \text { and } y \in D\right\} .
$$

Taking $\epsilon \rightarrow 0$ in (4.12) and (4.13), we finish the proof.

\section{ON ATTENUATION CORRECTION FOR CONVENTIONAL RECONSTRUCTION}

Since no inversion formula exists in the case when $\mu(x) \neq$ const, one frequently ignores attenuation and uses the classical Radon transform inversion formula

$$
\tilde{f}^{(\Phi)}(x)=\frac{1}{4 \pi^{2}} \int_{S^{1}} \int_{-\infty}^{\infty} \frac{\hat{f}_{, p}(\theta, p)}{\Theta \cdot x-p} d p d \theta
$$


for approximate inverting of the attenuated Radon transform data. Substituting (2.1) into (5.1), we get, similarly to (2.4),

$$
\tilde{f}^{(\Phi)}(x)=\frac{1}{(2 \pi)^{2}} \int_{\mathbb{R}^{2}} \int_{\mathbb{R}^{2}} b(y, \xi /|\xi|) f(y) e^{-i \xi \cdot(x-y)} d y d \xi .
$$

It is easy to see that the following analog of Theorem 1 holds.

Theorem 2. Under the assumptions of Theorem 1,

$$
\text { singsupp } \tilde{f}^{(\Phi)} \subset S \cup \Gamma_{D} \cup\left(\bigcup_{j} L_{j}\right) .
$$

Fix any $x_{0} \in S, x_{0} \notin \Gamma \cup\left(\bigcup_{j} L_{j}\right)$, such that $S$ is smooth in a neighborhood of $x_{0}$. Then

$$
\tilde{f}^{(\Phi)}(x)=\frac{b\left(x_{0}, n\left(x_{0}\right)\right)}{\pi} \operatorname{Im}\left\{\int_{0}^{\infty} \Psi_{f}(t, x) e^{i t h} d t\right\}, \quad x=x_{0}+h n\left(x_{0}\right),
$$

where $\Psi_{f}(t, x) \in C^{\infty}\left([0, \infty) \times U_{\epsilon}\left(x_{0}\right)\right)$ for some $\epsilon>0$. Moreover, $\Psi_{f}$ admits the asymptotic expansion

$$
\Psi_{f}(t, x) \sim \frac{D_{f}\left(x_{0}\right)}{t}+\sum_{k \geq 2} \frac{d_{k}(x)}{t^{k}}, \quad t \rightarrow \infty, d_{k} \in C^{\infty}\left(U_{\epsilon}\left(x_{0}\right)\right),
$$

which can be differentiated with respect to $t$ and $x$.

Fix any $x_{0} \in \Gamma_{D}, x \notin S \cup\left(\bigcup_{j} L_{j}\right)$. Then

$$
\tilde{f}^{(\Phi)}(x)=-\frac{A\left(x_{0}, \mu, f\right)}{2 \pi} \operatorname{Im}\left\{\int_{0}^{\infty} \Psi_{\mu}(t, x) e^{i t h} d t\right\}, \quad x=x_{0}+h n\left(x_{0}\right),
$$

where $\Psi_{\mu}(t, x) \in C^{\infty}\left([0, \infty) \times U_{\epsilon}\left(x_{0}\right)\right)$ for some $\epsilon>0$. Moreover, $\Psi_{\mu}$ admits the asymptotic expansion

$$
\Psi_{\mu}(t, x) \sim \frac{D_{\mu}\left(x_{0}\right)}{t}+\sum_{k \geq 2} \frac{d_{k}(x)}{t^{k}}, \quad t \rightarrow \infty, d_{k} \in C^{\infty}\left(U_{\epsilon}\left(x_{0}\right)\right),
$$

which can be differentiated with respect to $t$ and $x$.

Fix any $x_{0} \in S \cap \Gamma, x \notin \cup_{j} L_{j}$. Then

$$
\begin{aligned}
\tilde{f}_{\Lambda}^{(\Phi)}(x)= & \frac{b\left(x_{0}, n\left(x_{0}\right)\right)}{\pi} \operatorname{Im}\left\{\int_{0}^{\infty} \Psi_{f}(t, x) e^{i t h} d t\right\} \\
& -\frac{A\left(x_{0}, \mu, f\right)}{2 \pi} \operatorname{Im}\left\{\int_{0}^{\infty} \Psi_{\mu}(t, x) e^{i t h} d t\right\}, \\
x= & x_{0}+h n\left(x_{0}\right),
\end{aligned}
$$

where the functions $\Psi_{f}$ and $\Psi_{\mu}$ have the same properties as above.

Let us investigate the behavior of $\tilde{f}^{(\Phi)}$ in a neighborhood of $x_{0} \in S$. From (5.2) and (5.3), we get

$$
\begin{aligned}
\tilde{f}^{(\Phi)}(x) & \sim \frac{b\left(x_{0}, n\left(x_{0}\right)\right)}{\pi} D_{f}\left(x_{0}\right) \operatorname{Im}\left\{\int_{1}^{\infty} \frac{1}{t} e^{i t h} d t\right\} \\
& \sim b\left(x_{0}, n\left(x_{0}\right)\right) \frac{D_{f}\left(x_{0}\right)}{2} \operatorname{sgn}(h), \quad x=x_{0}+h n\left(x_{0}\right), h \rightarrow 0 .
\end{aligned}
$$


This implies that the value of the jump of $\tilde{f}^{(\Phi)}$ at $x_{0}$ equals $b\left(x_{0}, n\left(x_{0}\right)\right) D_{f}\left(x_{0}\right)$, which is different from the correct value $D_{f}\left(x_{0}\right)$. If the attenuation coefficient $\mu(x)$ is known, the value of the jump of $f$ can be recovered as follows.

1. Compute $\tilde{f}^{(\Phi)}$ and locate the discontinuity curve $S$ of $f$;

2. For a point $x_{0} \in S$, estimate $n\left(x_{0}\right)$;

3. Compute $b\left(x_{0}, n\left(x_{0}\right)\right)$ using (2.5); and

4. Divide the value of the jump of $\tilde{f}^{(\Phi)}$ at $x_{0}$ by $b\left(x_{0}, n\left(x_{0}\right)\right)$.

\section{Corollaries to Theorem 1 and Algorithmic implementation OF LOCAL TOMOGRAPHY}

Comparing Theorem 1 and the results in [K1] (see also Proposition 3 in Section 8 ), we see that the behavior of the local tomography function $\tilde{f}_{\Lambda}^{(\Phi)}$ in a neighborhood of $S=\operatorname{singsupp} f$ in the case of nonsmooth attenuation is basically the same as that in the case of smooth attenuation. Therefore we can use the results in [K1] for further analysis of the local tomography function $\tilde{f}_{\Lambda}^{(\Phi)}$ in the case of nonsmooth attenuation. These results are of a purely technical nature, and no proofs will be given. Interested readers are referred to [K1], where detailed proofs are presented.

Fix any $x_{0} \in S, x_{0} \notin \Gamma \cup\left(\bigcup_{j} L_{j}\right)$, such that $S$ is smooth in a neighborhood of $x_{0}$. According to $(2.9)$, the leading singular term of $\tilde{f}_{\Lambda}^{(\Phi)}$ in a neighborhood of $x_{0}$ is given by

$$
\begin{aligned}
\tilde{f}_{\Lambda}^{(\Phi)}(x) & \sim \frac{b\left(x_{0}, n\left(x_{0}\right)\right) D_{f}\left(x_{0}\right)}{\pi} \operatorname{Im}\left\{\int_{0}^{\infty} 1 \cdot e^{i t h} d t\right\}=\frac{b\left(x_{0}, n\left(x_{0}\right)\right) D_{f}\left(x_{0}\right)}{\pi} \frac{1}{h} \\
x & =x_{0}+h n\left(x_{0}\right), h \rightarrow 0
\end{aligned}
$$

Taking into account the second term in the asymptotic expansion of $\Psi_{f}$, we prove that

$$
\tilde{f}_{\Lambda}^{(\Phi)}(x)=\frac{b\left(x_{0}, n\left(x_{0}\right)\right) D_{f}\left(x_{0}\right)}{\pi} \frac{1}{h}+c_{1}(x) \ln |h|+c_{2}(x) \operatorname{sgn}(h)+c_{3}(x, h),
$$

where $c_{i}, i=1,2,3$, are smooth functions of $x \in U_{\epsilon}\left(x_{0}\right)$, and $c_{3}(x, h)$ satisfies the conditions

$$
c_{3}(x, h)=O(1), \frac{\partial}{\partial h} c_{3}(x, h)=O(\ln |h|), \quad h \rightarrow 0 .
$$

Numerically, instead of computing $\tilde{f}_{\Lambda}^{(\Phi)}$, we will compute its mollification

$$
\tilde{f}_{\Lambda \epsilon}^{(\Phi)}(x):=\left(W_{\epsilon} * \tilde{f}_{\Lambda}^{(\Phi)}\right)(x)=-\frac{1}{4 \pi} \int_{S^{1}} \int_{\mathbb{R}} w_{\epsilon}^{\prime \prime}(\Theta \cdot x-p) \hat{f}^{(\Phi)}(\theta, p) d p d \theta,
$$

where $w_{\epsilon}=R W_{\epsilon}$ is the classical Radon transform of $W_{\epsilon}$, and $W_{\epsilon}$ is a sequence of sufficiently smooth mollifiers with the properties

(a) $W_{\epsilon}(x)$ is a radial function, $W_{\epsilon}(x):=W_{\epsilon}(|x|)$;

(b) $W_{\epsilon}(x)=0,|x| \geq \epsilon$, and $W_{\epsilon}(x)>0,|x|<\epsilon$;

(c) $W_{\epsilon}(x)=\epsilon^{-2} W_{1}(x / \epsilon), \int_{|x| \leq 1} W_{1}(x) d x=1$; and

(d) $W_{\epsilon}(r)$ decreases on the interval $[0,1]$.

The following result describes the behavior of $\tilde{f}_{\Lambda \epsilon}^{(\Phi)}$ in a neighborhood of $x_{0} \in S$. 


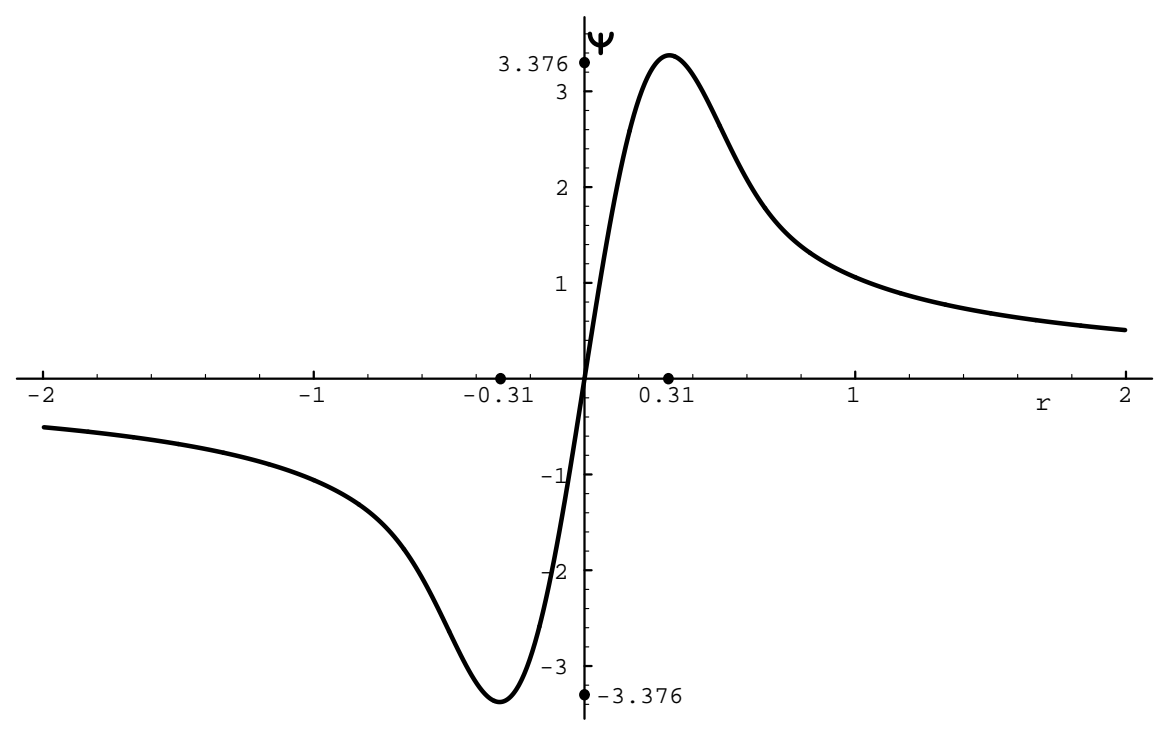

FIGURE 6.1. The graph of $\psi(r)$

\section{Theorem [K1].}

$$
\tilde{f}_{\Lambda \epsilon}^{(\Phi)}\left(x_{0}+\epsilon y\right)=\frac{b\left(x_{0}, n\left(x_{0}\right)\right) D_{f}\left(x_{0}\right)}{\pi} \frac{1+O(\epsilon)}{\epsilon} \psi\left(y \cdot n_{0}\right)+\psi_{\epsilon}\left(y \cdot n_{0}\right)+O(\epsilon \ln \epsilon),
$$

where $\psi_{\epsilon}(r)=O(\ln \epsilon)$ is an even function of $r$, and

$$
\left|\nabla \tilde{f}_{\Lambda \epsilon}^{(\Phi)}\left(x_{0}\right)\right|=\frac{b\left(x_{0}, n\left(x_{0}\right)\right) D_{f}\left(x_{0}\right)}{\pi} \frac{1+O(\epsilon)}{\epsilon^{2}} \psi^{\prime}(0),
$$

as $\epsilon \rightarrow 0$. In (6.5) and (6.6) it is assumed that $|y| \leq c<\infty$. The function $\psi(r)$ used in (6.5), (6.6) is odd and equals

$$
\psi(r)=\int_{r-1}^{r+1} \frac{w_{1}(r-t)}{t} d t .
$$

In the case of the mollifier

$$
W_{1}(x)=\frac{m+1}{\pi}\left(1-x^{2}\right)^{m}, \quad m=8,
$$

we have $\psi^{\prime}(0)=2(m+1)$, and the corresponding graph of $\psi$ is shown in Figure 6.1 .

Now let us consider an algorithm for finding values of jumps of $f$. We will describe the algorithm which works in the $x$-domain with the already computed local tomography function $\tilde{f}_{\Lambda \epsilon}^{(\Phi)}(x)$. This algorithm will be based on equation (6.5). An algorithm which computes the gradient of $\tilde{f}_{\Lambda \epsilon}^{(\Phi)}$ in one step and then uses equation (6.6) for finding values of jumps of $f$ can also be developed (see [K1]).

Let us assume that values of $\tilde{f}_{\Lambda \epsilon}^{(\Phi)}$ are calculated on a square grid with step size $h: x_{i j}=\left(x_{i}^{(1)}, x_{j}^{(2)}\right)=(i h, j h), i, j \in \mathbb{Z}$. Let us choose a grid node $x_{i_{0} j_{0}}$ on $S$. Let 
us suppose that $h$ and $\epsilon$ are sufficiently small. Then we can rewrite (6.5) as follows:

$$
\tilde{f}_{\Lambda \epsilon}^{(\Phi)}(x) \approx \frac{b\left(x_{0}, n\left(x_{0}\right)\right) D_{f}\left(x_{0}\right)}{\pi \epsilon} \psi\left(\frac{x-x_{0}}{\epsilon} \cdot n_{0}\right)+\psi_{\epsilon}\left(\frac{x-x_{0}}{\epsilon} \cdot n_{0}\right), \quad x_{0}=x_{i_{0} j_{0}} .
$$

Fix $n_{1}, n_{2} \in \mathbb{N}$ and consider a $\left(2 n_{1}+1\right) \times\left(2 n_{2}+1\right)$ window around $x_{i_{0} j_{0}}$. To use (6.9) for finding $D_{f}\left(x_{i_{0} j_{0}}\right)$, first we need to estimate $n_{0}$. This can be easily done by computing partial derivatives:

$$
N_{0} \approx \frac{\left(\tilde{f}_{\Lambda \epsilon}^{(\Phi)}\left(x_{i_{0}+1, j_{0}}\right)-\tilde{f}_{\Lambda \epsilon}^{(\Phi)}\left(x_{i_{0}-1, j_{0}}\right), \tilde{f}_{\Lambda \epsilon}^{(\Phi)}\left(x_{i_{0}, j_{0}+1}\right)-\tilde{f}_{\Lambda \epsilon}^{(\Phi)}\left(x_{i_{0}, j_{0}-1}\right)\right)}{\sqrt{\left(\tilde{f}_{\Lambda \epsilon}^{(\Phi)}\left(x_{i_{0}+1, j_{0}}\right)-\tilde{f}_{\Lambda \epsilon}^{(\Phi)}\left(x_{i_{0}-1, j_{0}}\right)\right)^{2}+\left(\tilde{f}_{\Lambda \epsilon}^{(\Phi)}\left(x_{i_{0}, j_{0}+1}\right)-\tilde{f}_{\Lambda \epsilon}^{(\Phi)}\left(x_{i_{0}, j_{0}-1}\right)\right)^{2}}} .
$$

Since $\psi(r)$ is odd and $\psi_{\epsilon}(r)$ is even in $r$, we have

$$
D_{f}\left(x_{i_{0} j_{0}}\right) \approx \frac{\pi \epsilon}{b\left(x_{i_{0} j_{0}}, N_{0}\right)} \frac{\sum_{\substack{\left|i-i_{0}\right| \leq n_{1} \\\left|j-j_{0}\right| \leq n_{2}}} \tilde{f}_{\Lambda \epsilon}^{(\Phi)}\left(x_{i j}\right) \psi\left(\frac{x_{i j}-x_{i_{0} j_{0}}}{\epsilon} \cdot N_{0}\right)}{\sum_{\substack{i-i_{0}\left|\leq n_{1}\\\right| j-j_{0} \mid \leq n_{2}}} \psi^{2}\left(\frac{x_{i j}-x_{i_{0} j_{0}}}{\epsilon} \cdot N_{0}\right)} .
$$

Equations (6.1) and (6.5) imply that the larger values of $\tilde{f}_{\Lambda \epsilon}^{(\Phi)}$ correspond to the side of $S$ with the larger values of $f$. Thus, we came to the following algorithm for estimating values of jumps of $f$ from $\tilde{f}_{\Lambda \epsilon}^{(\Phi)}$ :

1. Estimate the vector $N_{0}$ by (6.10) and compute $b\left(x_{i j}, N_{0}\right)$ using (2.5);

2. Compute the estimate of $D_{f}\left(x_{i j}\right)$ by $(6.11)$; and

3. The vector $N_{0}$ given by (6.10) points from the smaller values of $f$ to the larger values of $f$.

Suppose now that $\operatorname{supp} \mu \subsetneq D=\operatorname{supp} f$ and there exists $x_{0} \in \Gamma$ such that $f \in C^{\infty}\left(U_{\epsilon}\left(x_{0}\right)\right)$ and $L_{\Gamma}\left(x_{0}\right) \cap \operatorname{supp} \mu=\left\{x_{0}\right\}$. Here $L_{\Gamma}\left(x_{0}\right)$ is the line tangent to $\Gamma$ at $x_{0}$. In this case we can find the value of the jump of $\mu$ across $\Gamma$ at $x_{0}$. Since $\mu=0$ on $L_{\Gamma}\left(x_{0}\right)$, we have

$$
\int_{t}^{\infty} \mu\left(x_{0}+s n\left(x_{0}\right)^{\perp}\right) d s=\int_{-\infty}^{t} \mu\left(x_{0}+s n\left(x_{0}\right)^{\perp}\right) d s=0, \quad t \in \mathbb{R} .
$$

Therefore,

$$
A\left(x_{0}, \mu, f\right)=\hat{f}\left(n\left(x_{0}\right), x_{0} \cdot n\left(x_{0}\right)\right)=\hat{f}^{(\Phi)}\left(n\left(x_{0}\right), x_{0} \cdot n\left(x_{0}\right)\right) .
$$

To improve the accuracy of computing $A\left(x_{0}, \mu, f\right)$ in the case of noisy data, we can use the formula

$$
A\left(x_{0}, \mu, f\right)=\frac{1}{2}\left[\hat{f}^{(\Phi)}\left(n\left(x_{0}\right), x_{0} \cdot n\left(x_{0}\right)\right)+\hat{f}^{(\Phi)}\left(-n\left(x_{0}\right),-x_{0} \cdot n\left(x_{0}\right)\right] .\right.
$$

Note that $A\left(x_{0}, \mu, f\right)$ can be computed using only the data which is available. Comparing equations (2.9) and (2.11), we see that the jump of $\mu$ at $x_{0}$ can be computed using the same algorithm as the one for finding jumps of $f$, but instead 

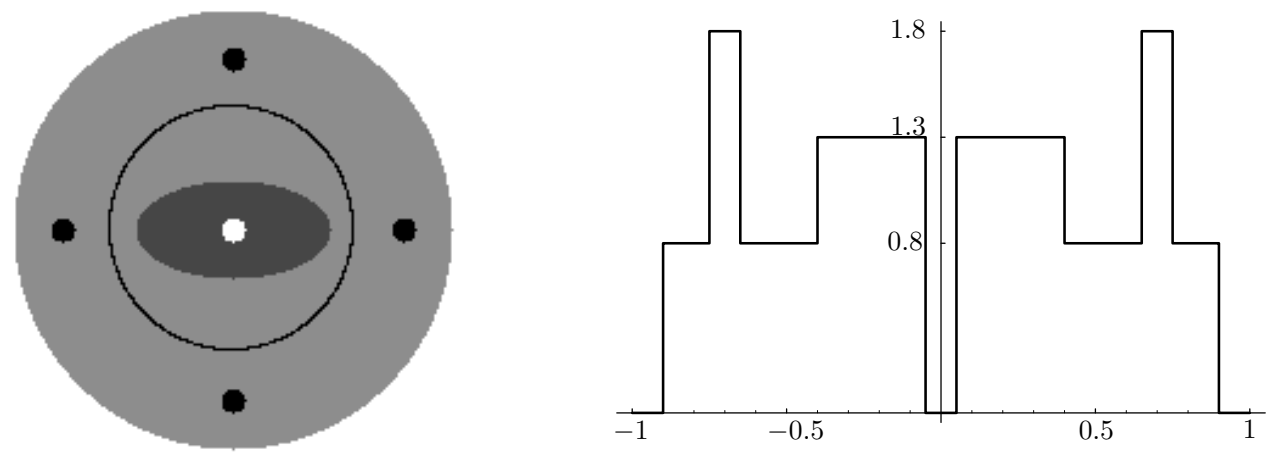

Figure 7.1. Phantom $f$ used for generating the attenuated Radon transform data (left panel) and central horizontal cross-section of $f$ (right panel).

of (6.11) we will use the formula

$$
D_{\mu}\left(x_{i_{0} j_{0}}\right) \approx-\frac{2 \pi \epsilon}{A\left(x_{i_{0} j_{0}}, \mu, f\right)} \frac{\sum_{\substack{\left|i-i_{0}\right| \leq n_{1} \\\left|j-j_{0}\right| \leq n_{2}}} \tilde{f}_{\Lambda \epsilon}^{(\Phi)}\left(x_{i j}\right) \psi\left(\frac{x_{i j}-x_{i_{0} j_{0}}}{\epsilon} \cdot N_{0}\right)}{\sum_{\substack{\left|i-i_{0}\right| \leq n_{1} \\\left|j-j_{0}\right| \leq n_{2}}} \psi^{2}\left(\frac{x_{i j}-x_{i_{0} j_{0}}}{\epsilon} \cdot N_{0}\right)},
$$

where $A\left(x_{i_{0} j_{0}}, \mu, f\right)$ can be computed by (6.12) with $n\left(x_{0}\right)=N_{0}$.

\section{Results OF NUmerical testing}

In Figure 7.1, left panel, we see the phantom used for generating the attenuated Radon transform data. The densities are as follows - exterior: 0, ellipse: 1.3, area outside the ellipse: 0.8 , four small disks off the center: 1.8 , the small disk at the center: 0 . The radius of the phantom: 0.9, the half-axes of the ellipse: 0.2 and 0.4 , the radii of the four small discs: 0.05 . The circle outside the ellipse denotes the discontinuity curve $\Gamma$ of $\mu$. The central horizontal cross-section of the density function $f$ is shown in Figure 7.1, right panel. The tomographic data were computed for 350 angles equispaced on $[0,2 \pi)$ and 601 projections per angle.

The density plot of the mollified local tomography function $\tilde{f}_{\Lambda \epsilon}^{(\Phi)}$ is shown in Figure 7.2 , left and right panels. The attenuation coefficient in this example is given by $\mu(x)=0$ if $|x|>0.5$ and $\mu(x)=1$ if $|x| \leq 0.5$. The singular support of $\mu$ shows up in the figure in the same fashion as the singular support of $f$ (which is in agreement with Theorem 1). In this and the following examples, values of the local tomography function have been computed at the nodes of a square $201 \times 201$ grid with step size $h=0.01$. As a mollifier, we used the function defined in (6.8) with the radius of mollification $\epsilon=0.03$.

The left and right panels of Figure 7.2 depict the same function $\tilde{f}_{\Lambda \epsilon}^{(\Phi)}$, but the grey level scale was adjusted in the right panel to make the lines $L_{j} \in \operatorname{singsupp} \tilde{f}_{\Lambda}^{(\Phi)}$ visible. Recall that $L_{j}$ are the lines which satisfy conditions (a)-(c) in Theorem 

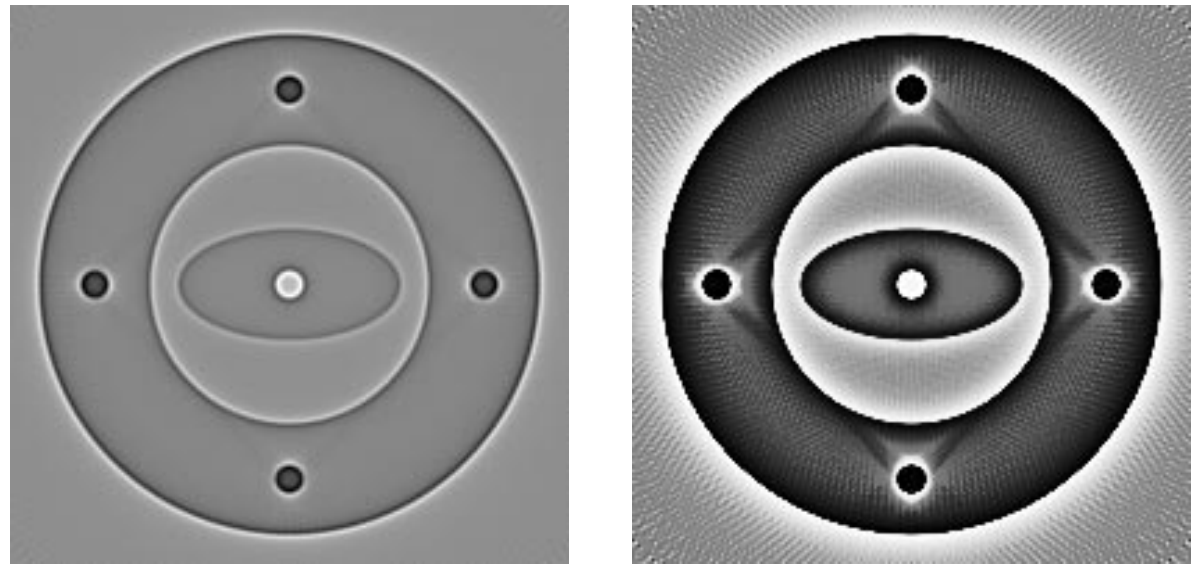

Figure 7.2. Density plot of the local tomography function $\tilde{f}_{\Lambda \epsilon}^{(\Phi)}$. The attenuation coefficient is given by $\mu(x)=0$ if $|x|>0.5$ and $\mu(x)=1$ if $|x| \leq 0.5$.

1. The lines $L_{j}$ cannot be seen in the left panel. This indicates that singularity of $\tilde{f}_{\Lambda}^{(\Phi)}$ is weaker in neighborhoods of $L_{j}$ than in neighborhoods of $S$ and $\Gamma$.

The density plots of $D_{f}$ and $D_{\mu}$, estimated by (6.11) and (6.13), are shown in Figure 7.3. Only the area $|x|<0.8$ is shown in the bottom panel, because $\hat{f}^{(\Phi)}$ is relatively small when $|x|>0.8$ and dividing by $\hat{f}^{(\Phi)}$ results in large numerical values. Central horizontal cross-sections of $D_{f}$ and $D_{\mu}$ are shown in Figure 7.4. In the top panel, the line with peaks is the graph of the estimated $D_{f}(x)$, and the big dots represent positions and amplitudes of jumps of the original density function $f$ (cf. Figure 7.1). In the bottom panel, the line with peaks is the graph of the estimated $D_{\mu}$, and the big dots represent positions and amplitudes of jumps of $\mu$. In both panels we see a good agreement between the dots and the maxima of the peaks.

In Figure 7.5 , left panel, we see the density plot of $\tilde{f}_{\Lambda \epsilon}^{(\Phi)}$ computed in the case of an attenuation coefficient given by $\mu(x)=0.3$ if $0.5<|x|<0.9$ and $\mu(x)=1$ if $|x| \leq 0.5$. The density plot of the estimated $D_{f}$ is shown in Figure 7.5, right panel. The central horizontal cross-section of $D_{f}$ is shown in Figure 7.6.

Fix any $x_{0}$ on the boundary of $\operatorname{supp} f$, i.e. $\left|x_{0}\right|=0.9$. Since $f=0$ on any line tangent to $\partial D$, equation (2.12) implies $A\left(x_{0}, \mu, f\right)=0$. Therefore, (2.14) and (2.9) are identical in this case. This shows that if $f$ has a jump across $\partial D$, then the values of the jump can be found using (6.11), and the attenuation coefficient can be ignored. The results shown in Figure 7.6 confirm the conclusion.

\section{Auxiliary Results needed for the Proof of Theorem 1}

Proposition 1 [RK, p.121]. Consider the ray $\left\{x(t)=y_{0}+t \Theta_{0}^{\perp}, t \geq 0\right\}$ such that $y_{0} \notin \Gamma$ and $x(t)$ is transversal to $\Gamma$. Then $X(y, \theta)=\int_{0}^{\infty} \mu\left(y+t \Theta^{\perp}\right) d t$ is a smooth function in a neighborhood of $\left(y_{0}, \theta_{0}\right)$. 

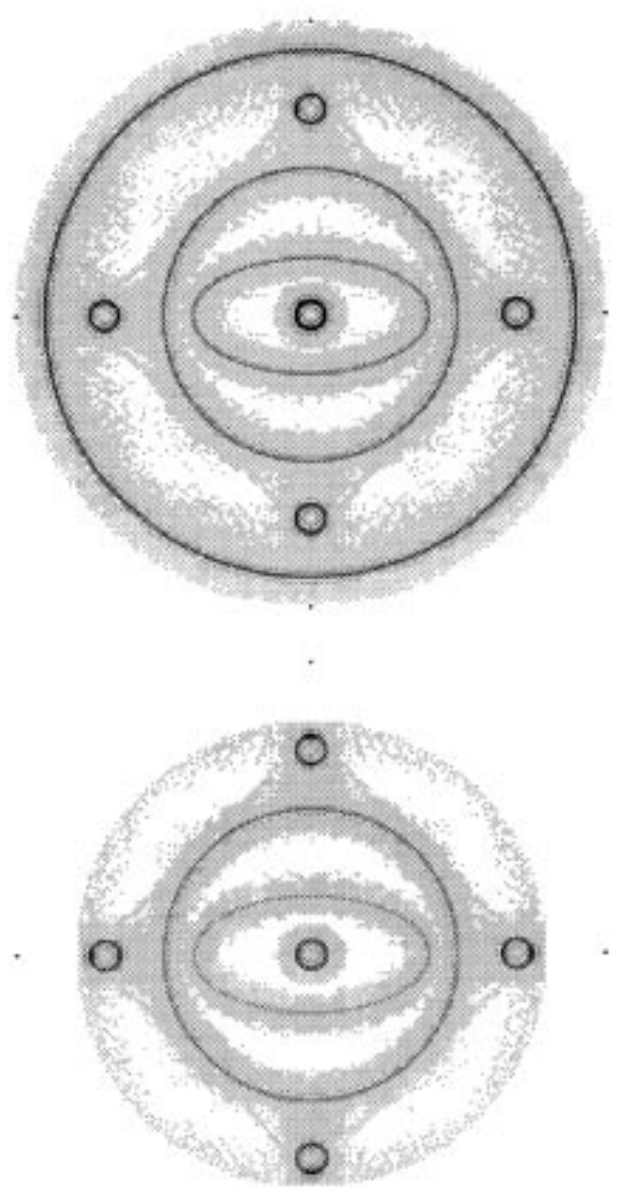

Figure 7.3. Density plots of the estimated $D_{f}(x)$ (top panel) and $D_{\mu}(x)$ (bottom panel).

Proof. We have

$$
X(y, \theta)=\sum_{k=0}^{N} \int_{t_{k}(y, \theta)}^{t_{k+1}(y, \theta)} \mu\left(y+t \Theta^{\perp}\right) d t,
$$

where $x\left(t_{k}(y, \theta)\right), k \geq 1$, are points of intersection of the ray $\left\{x(t)=y+t \Theta^{\perp}, t \geq 0\right\}$ and $\Gamma$, and $t_{0}(y, \theta) \equiv 0$. Since the ray $\left\{y_{0}+t \Theta_{0}^{\perp}, t \geq 0\right\}$ is transversal to $\Gamma$ and $\Gamma$ is smooth, the implicit function theorem yields that the functions $t_{k}(y, \theta)$ are smooth in a sufficiently small neighborhood of $\left(y_{0}, \theta_{0}\right)$. Since $\mu\left(y+t \Theta^{\perp}\right) \in$ $C^{\infty}\left(\left[t_{k}(y, \theta), t_{k+1}(y, \theta)\right]\right), k \geq 0$, the desired assertion follows immediately from (8.1). 

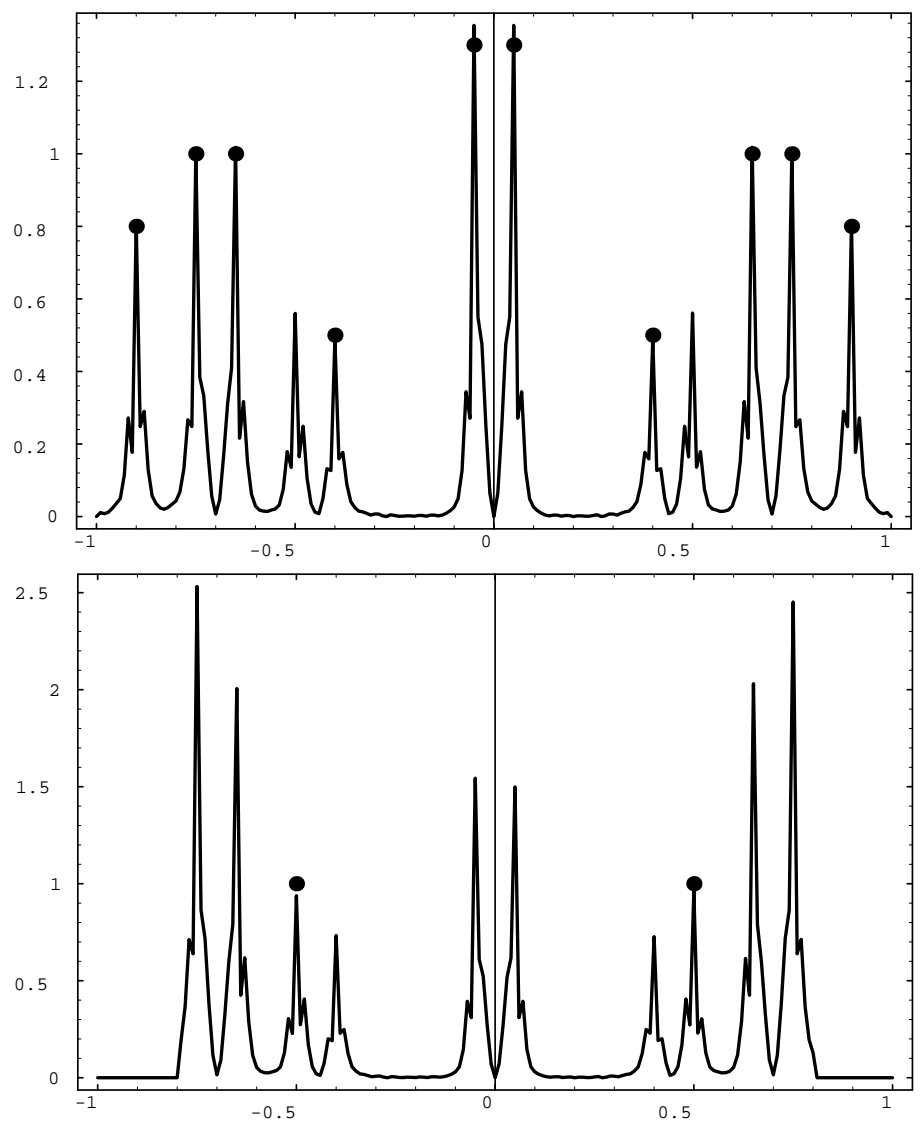

Figure 7.4. Central horizontal cross-sections of the estimated $D_{f}$ (top panel) and $D_{\mu}$ (bottom panel).

\section{Proposition 2.}

$$
\begin{gathered}
(\eta(\theta, p)+\nu(\theta, p)) / 2=\psi(\theta, p), \\
\Theta \in \Omega, p \leq p_{0}(\theta), \psi(\theta, p) \in C^{\infty}\left(\Omega \times\left[p_{0}(\theta)-\epsilon, p_{0}(\theta)\right]\right) .
\end{gathered}
$$

Proof. As in the proof of Lemma 1, let $t=\eta(\theta, p)$ and $t=\nu(\theta, p)$ be the points of intersection of the line $\left\{p \Theta+t \Theta^{\perp}, t \in \mathbb{R}\right\}$ and $\Gamma$. Fix any $\Theta \in \Omega$ and let $x_{0}(\theta) \in \Gamma$ be a point such that $n\left(x_{0}\right)=\Theta$. Introduce the coordinate system with the origin at $x_{0}$ and $x_{1}$-axis pointing in the direction $\Theta$. A local equation of $\Gamma$ in the new coordinate system is given by $u=\varphi_{x_{0}}(v)$, where $\varphi_{x_{0}}(0)=\varphi_{x_{0}}^{\prime}(0)=0$ and $\varphi_{x_{0}}^{\prime \prime}(0) \neq 0$. Suppose, for example, that $\varphi_{x_{0}}^{\prime \prime}(0)<0$; that is, $\Theta$ points away from the center of curvature of $\Gamma$ at $x_{0}$. By the Morse lemma [GuSt, p.16], there exists a diffeomorphism $g_{x_{0}}$ such that $v=g_{x_{0}}(s)$ and $u=\varphi_{x_{0}}\left(g_{x_{0}}(s)\right)=-s^{2}$. Fix $p<0$ such that $|p|$ is sufficiently small. Solving the equations $p \Theta+t \Theta^{\perp}=\left(\varphi_{x_{0}}(v), v\right)$ with respect to $t$, we find that $p=-s^{2}$ and $t=g_{x_{0}}(s)$; that is, $t_{ \pm}(p)=g_{x_{0}}( \pm \sqrt{-p}), p \leq$ 0 . Expanding $g_{x_{0}}(s)$ in a Taylor series about $s=0$, collecting the terms with even and odd powers of $s$, and factoring out $\sqrt{-p}$, we get

$$
t_{ \pm}(p)= \pm g_{1, x_{0}}(p) \sqrt{-p}+g_{2, x_{0}}(p), p \leq 0,
$$



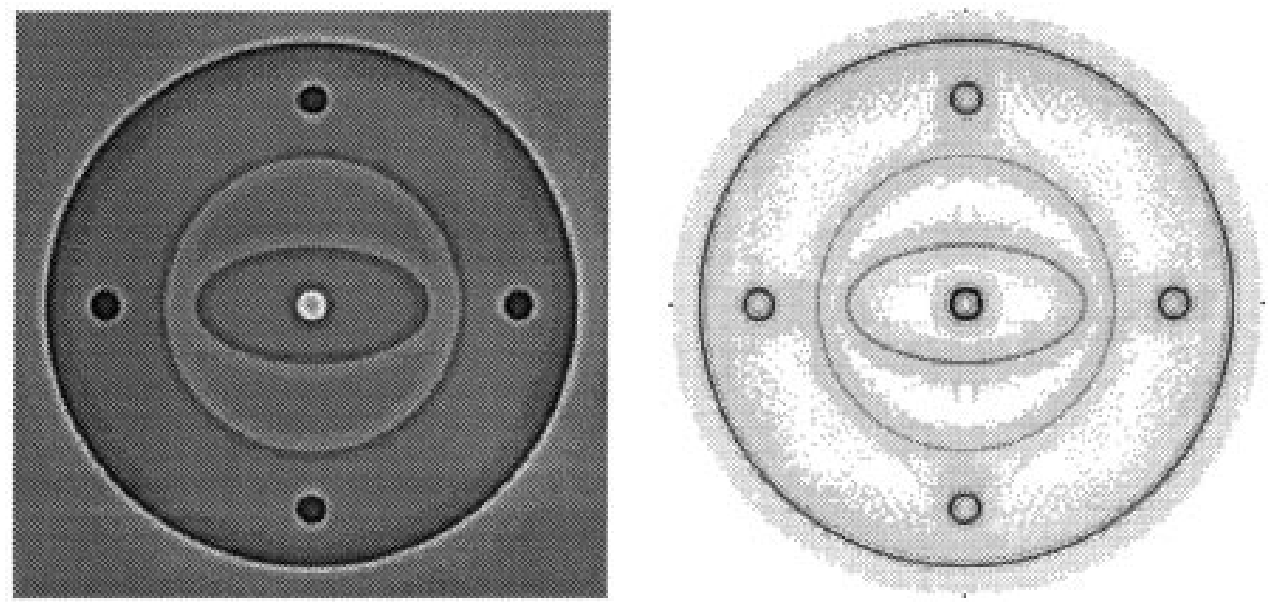

Figure 7.5. Density plots of $\tilde{f}_{\Lambda \epsilon}^{(\Phi)}$ (left panel) and $D_{f}$ (right panel). The attenuation coefficient is given by $\mu(x)=0.3$ if $|x|>0.5$ and $\mu(x)=1$ if $|x| \leq 0.5$.

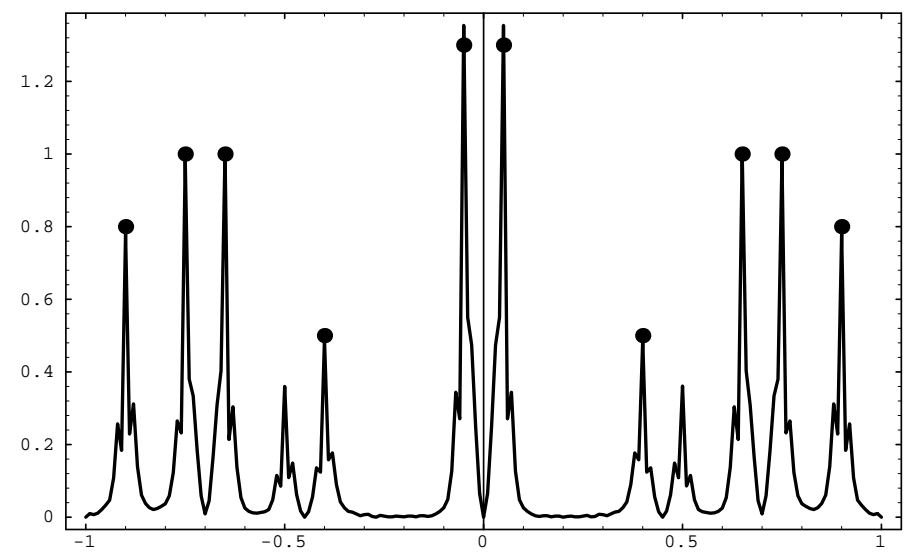

Figure 7.6. Central horizontal cross-section of $D_{f}$ corresponding to Figure 7.5 (right panel).

where $g_{k, x_{0}}(p), k=1,2$, are smooth functions of $p$ in a neighborhood of $p=0$. Since the radius of curvature of $\Gamma$ is finite, one can easily show, using the implicit function theorem, that $x_{0}(\theta)$ is a smooth function of $\theta$. Since the local equation of $\Gamma, u=\varphi_{x_{0}}(v)$, depends smoothly on $x_{0}$, we conclude that $\varphi_{x_{0}}$ depends smoothly on $\theta$. This implies that the diffeomorphisms $v=g_{x_{0}}(s)$ also depend smoothly on $\theta$ [GuSt, p.17]. Moreover, the matrices of transformation from the temporary coordinate systems, related to $x_{0}(\theta), \Theta$, back to the original coordinate system depend smoothly on $\theta$. According to $(8.2),\left(t_{-}(p)+t_{+}(p)\right) / 2$ is a smooth function of $p \leq 0$ and $x_{0}$, and the desired assertion follows immediately. 
Finally, for convenience of the reader, we will present the result of [K1], which was used in the proof of Lemma 2. The following is an immediate corollary to Theorem 2.1 in [K1] or to Theorem 18.2.12 in [Hor], which describes how PDO act on conormal distributions.

Proposition 3. Let a $P D O \mathcal{B} \in C L^{\gamma}\left(\mathbb{R}^{n}\right)$ be a classical PDO with an amplitude $B(x, y, \xi)$ :

$$
\begin{gathered}
B(x, y, \xi) \sim \sum_{k \geq 0} b_{k}(x, y, \Theta) t^{\gamma-k} \\
\gamma \in \mathbb{R}, b_{k}(x, y, \Theta) \in C^{\infty}\left(\mathbb{R}^{n} \times \mathbb{R}^{n} \times S^{n-1}\right), t=|\xi|, \Theta=\xi /|\xi| .
\end{gathered}
$$

Suppose that $B(x, y, \xi)$ is even in $\xi: B(x, y, \xi)=B(x, y,-\xi)$. Fix a sufficiently small open set $U \subset \mathbb{R}^{n}$ such that $S \cap U \neq \varnothing, S$ is smooth inside $U$, and $b_{0}\left(x, x, n\left(x_{0}\right)\right)$ $\neq 0$ for $x \in U, x_{0} \in S \cap U$. Then one has

$$
\begin{gathered}
(\mathcal{B} f)(x)=\frac{b_{0}\left(x, x, n\left(x_{0}\right)\right)}{\pi} \operatorname{Im}\left\{\int_{0}^{\infty} \Psi(t, x) e^{i t h} d t\right\}, \\
x=x_{0}+h n\left(x_{0}\right) \in U, x_{0} \in S \cap U,
\end{gathered}
$$

where $\Psi(t, x) \in C^{\infty}([0, \infty) \times U)$. Moreover, $\Psi$ admits the asymptotic expansion

$$
\Psi(t, x) \sim t^{\gamma-1}\left(D_{f}\left(x_{0}\right)+\sum_{k \geq 1} \frac{d_{k}(x)}{t^{k}}\right), \quad t \rightarrow \infty, d_{k} \in C^{\infty}(U),
$$

which can be differentiated with respect to $t$ and $x$.

\section{REFERENCES}

[BR] M. Beals and M. Reed, Microlocal regularity theorems for nonsmooth pseudodifferential operators and applications to nonlinear problems, Trans. Amer. Math. Soc. 285 (1) (1984), 159-184. MR 86a:35156

[BW] C.A. Berenstein and D.F. Walnut, Local inversion of the Radon transform in even dimensions using wavelets, 75 Years of Radon Transform (S. Gindikin and P. Michor, eds.), Conference Proceedings and Lecture Notes in Mathematical Physics, Vol. 4, International Press, Boston, 1994, pp. 45-69. MR 95m:42037

[Be] G. Beylkin, The inversion problem and applications of the generalized Radon transform, Comm. on Pure and Appl. Math. 37 (1984), 579-599. MR 86a:44002

[BH] N. Bleistein and R. Handelsman, Asymptotic Expansions of Integrals, Dover, Mineola, N.Y., 1986. MR 89d:41049

[De] S. Deans, The Radon Transform and Some of Its Applications, Wiley, New York, 1983. MR 86a: 44003

[FRS] A. Faridani, E.L. Ritman, and K.T. Smith, Local tomography, SIAM J. Appl. Math. 52 (2) (1992), 459 - 484, 1193 - 1198. MR 93b:92008; CMP 92:15

[FFRS] A. Faridani, D. Finch, E.L. Ritman, and K.T. Smith, Local tomography II, SIAM J. Appl. Math. 57 (1997), 1095-1127. CMP 97:16

[GS] I.M. Gelfand and G.E. Shilov, Generalized Functions, Volume I, Academic Press, New York, 1964. MR 29:3869

[GuSt] V. Guillemin and S. Sternberg, Geometric Asymptotics, Amer. Math. Soc., Providence, RI, 1977. MR 58:24404

[GU] V. Guillemin and G. Uhlmann, Oscillatory integrals with singular symbols, Duke Mathematical Journal 48 (1981), 251-267. MR 28d:58065

[Hor] L. Hörmander, The Analysis of Linear Partial Differential Operators. III, SpringerVerlag, Berlin, 1985. MR 87d:35002a

[K1] A.I. Katsevich, Local tomography for the generalized Radon transform, SIAM J. Appl. Math. 57 (4) (1997), 1128-1162. CMP 97:16 
[K2] Local tomography for the limited-angle problem, J. Math. Anal. Appl. 213 (1997), 160-182. CMP 98:01

[KR1] A.I. Katsevich and A.G. Ramm, Asymptotics of PDO on discontinuous functions near singular support, Applicable Analysis 58 (3-4) (1995), 383-390. MR 97d:35259

[KR2] Finding jumps of a function using local tomography, PanAmerican Mathematical Journal 6 (2) (1996), 1-21. MR 97e:44004

[KR3] - New methods for finding values of the jumps of a function from its local tomographic data, Inverse Problems 11 (5) (1995), 1005-1023. CMP 96:02

[KLM] P. Kuchment, K. Lancaster, and L. Mogilevskaya, On local tomography, Inverse Problems 11 (1995), 571-589. MR 96i:44007

[KS] P. Kuchment and I. Shneiberg, Some inversion formulas in the single photon emission computed tomography, Applicable Analysis 53 (1994), 221-231. MR 96m:44003

[Kun] L.A. Kunyansky, Generalized and attenuated Radon transforms: restorative approach to the numerical inversion, Inverse Problems 8 (1992), 809-819. MR 93k:65103

[Ma1] J. Marschall, Parametrices for nonregular elliptic pseudodifferential operators, Math. Nachr. 159 (1992), 175-188. MR 94h:35287

[Ma2] On the boundedness and compactness of nonregular pseudo-differential operators, Math. Nachr. 175 (1995), 231-262. MR 96k:47090

[MU] R. Melrose and G. Uhlmann, Lagrangian intersection and the Cauchy problem, Comm. Pure Appl. Math. 32 (1979), 483-519. MR 81b:58052

[R1] A.G. Ramm, Optimal local tomography formulas, PanAmer. Math. J. 4 (4) (1994), 125127. MR 95h: 44008

[R2] _ Finding discontinuities from tomographic data, Proc. Amer. Math. Soc. 123 (8) (1995), 2499-2505. MR 95j:44001

[R3] Necessary and sufficient conditions for a PDO to be a local tomography operator, Comptes Rend. Acad. Sci, Paris, Sér. I Math. 332 (7) (1996), 613-618. MR 97g:47046

[RK] A.G. Ramm and A.I. Katsevich, The Radon Transform and Local Tomography, CRC Press, Boca Raton, FL, 1996. MR 97g:44009

[RZ1] A.G. Ramm and A.I. Zaslavsky, Singularities of the Radon transform, Bull. Amer. Math. Soc. 25 (1) (1993), 109-115. MR 93i:44003

[RZ2] , Reconstructing singularities of a function its Radon transform, Math. and Comput. Modelling 18 (1) (1993), 109 - 138. MR 94j:44006

[Shu] M.A. Shubin, Pseudodifferential Operators and Spectral Theory, Springer-Verlag, Berlin, 1987. MR 88c: 47105

[SK] K.T. Smith and F. Keinert, Mathematical foundations of computed tomography, Appl. Optics (1985), $3950-3957$.

[Ta] M.E. Taylor, Pseudodifferential Operators and Nonlinear PDE, Progress in Math., Vol. 100, Birkhauser, Boston, MA, 1991. MR 92j:35193

[TM] O.J. Tretiak and C.L. Metz, The exponential Radon transform, SIAM J. Appl. Math. 39 (1980), 341 - 354. MR 82a: 44004

[VKK] E.I. Vainberg, I.A. Kazak, and V.P. Kurczaev, Reconstruction of the internal threedimensional structure of objects based on real-time integral projections, Soviet J. Nondest. Test. 17 (1981), 415-423.

[W] R. Wong, Asymptotic Approximations of Integrals, Academic Press, Boston, 1989. MR 90j:41061

Los Alamos National Laboratory, MS K-990, Los Alamos, New Mexico 87545

Current address: Department of Mathematics, University of Central Florida, Orlando, Florida 32816-1364

E-mail address: akatsevi@pegasus.cc.ucf.edu 